\title{
High-temperature conventional superconductivity in the boron-carbon system: Material trends
}

\author{
Santanu Saha $\odot,{ }^{1, *}$ Simone Di Cataldo $\odot,{ }^{1,2}$ Maximilian Amsler, ${ }^{3,4}$ Wolfgang von der Linden, ${ }^{1}$ and Lilia Boeri ${ }^{2}$ \\ ${ }^{1}$ Graz University of Technology, NAWI Graz, 8010 Graz, Austria \\ ${ }^{2}$ Dipartimento di Fisica, Università di Roma La Sapienza, Piazzale Aldo Moro 5, I-00185 Roma, Italy \\ ${ }^{3}$ Department of Materials Science and Engineering, Cornell University, Ithaca, New York 14853, USA \\ ${ }^{4}$ Department of Chemistry and Biochemistry, University of Bern, Freiestrasse 3, CH-3012 Bern, Switzerland
}

(Received 10 April 2020; revised 29 June 2020; accepted 30 June 2020; published 23 July 2020)

\begin{abstract}
In this paper, we probe the possibility of high-temperature conventional superconductivity in the boron-carbon system, using $a b$ initio screening. A database of 320 metastable structures with fixed composition (50\%/50\%) is generated with the minima hopping method, and characterized with electronic and vibrational descriptors. Full electron-phonon calculations on 16 representative structures allow us to identify general trends in $T_{c}$ across and within the four families in the energy landscape, and to construct an approximate $T_{c}$ predictor, based on transparently interpretable and easily computable electronic and vibrational descriptors. Based on these, we estimate that around $10 \%$ of all metallic structures should exhibit $T_{c}$ 's above $30 \mathrm{~K}$. This paper is a first step toward $a b$ initio design of new high- $T_{c}$ superconductors.
\end{abstract}

DOI: 10.1103/PhysRevB.102.024519

\section{INTRODUCTION}

For more than a century, the discovery of a roomtemperature superconductor has been considered one of the holy grails of condensed-matter physics. At the end of the '60s, Ashcroft and Ginzburg [1,2] predicted that under sufficiently high pressures, hydrogen may be turned into an atomic metallic phase [3] which would behave as a hightemperature superconductor (HTS). However, until recently, the pressures involved in hydrogen metallization turned out to be prohibitive, even for the best high-pressure research labs worldwide [4-6].

In 2004, Ashcroft proposed that the metallization pressure may be sensibly reduced by exploiting chemical precompression of the hydrogen sublattice in H-rich compounds. $\mathrm{SH}_{3}$ [7], predicted in 2014 by Duan et al. [8], was experimentally found to be superconducting at $200 \mathrm{GPa}$ with a $T_{c}=203 \mathrm{~K}$ by the Eremets group [9]; in less than five years, the maximum $T_{c}$ in high-pressure hydrides was raised to $265 \mathrm{~K}$ in $\mathrm{LaH}_{10}$, predicted a few years before [10-13].

Although room-temperature superconductivity at high pressures is an impressive achievement by itself, practical applications of superconductivity require materials that can operate at ambient pressure. Thus, the main focus of superconductivity research is gradually shifting from roomtemperature superconductivity at high pressures to HTSs at ambient pressure [14].

Proposals to realize HTSs at ambient pressure based on the conventional electron-phonon ( $e p$ ) mechanism-high- $T_{c}$ conventional superconductivity (HTCS) — date back to the early 2000s, when the $\mathrm{MgB}_{2}$ discovery [15] showed that HTCS is best realized in covalent metals [16], where the high-phonon frequencies and strong $e p$ matrix elements typical of covalent

\footnotetext{
*santanu.saha@tugraz.at
}

bonds coexist with metallic behavior, which is a prerequisite for conventional superconductivity [17-21]. Following this general principle, several hypothetical materials were proposed, such as doped LiBC, graphane, etc. [22,23]. These are all chemical and structural analogs of $\mathrm{MgB}_{2}$ proposed on the basis of simple physical arguments but without a knowledge of the underlying thermodynamics. A notable exception was hexagonal $\mathrm{Li}-\mathrm{B}$, which was shown to be thermodynamically stable and synthesized [24-26] although its $T_{c}$ is predicted to be much lower than that of $\mathrm{MgB}_{2}$.

Only recently, the widespread use of modern methods for crystal-structure prediction (CSP) has permitted us to address the crucial aspect of thermodynamics in material design.

Combined with methods for high-throughput database (DB) screening and machine learning, CSP methods are an unprecedentedly powerful tool driving a sudden acceleration in material discoveries recently $[4,27,28]$. However, compared to other problems of material research, their application to superconductivity is still at a very early stage [29-32] due to two intrinsic problems: (i) for a large class of unconventional superconductors, including the high- $T_{c}$ cuprates, a quantitative theory of superconductivity is currently missing and (ii) for conventional superconductors where, on the other hand, $T_{c}$ can be predicted with quantitative accuracy, the cost of a single $T_{c}$ calculation is too high to directly perform highthroughput screening of large DBs of hypothetical materials [14].

This paper is part of a larger project in which we plan to investigate superconductivity trends across several families of conventional superconductors to identify meaningful screening protocols to search for promising superconducting candidates. In this paper, we focus on boron-carbon (BC) structures, with a 50\%-50\% composition.

First, we generate a large DB of 320 distinct metastable boron/carbon structures with the minima hopping method (MH) [33-35]. The whole set is then analyzed to identify the 
main structural templates characterizing the potential energy surface; on the basis of simple electronic and vibrational descriptors, the number of structures is progressively narrowed down to a set of 16 representative structures, for which we perform full $T_{c}$ calculations, to identify and understand empirical trends governing superconductivity in BC systems.

The BC system is an ideal choice for a first blind study of superconductivity because both boron and carbon are light elements which tend to form stiff, directional bonds, favorable for HCTS; furthermore, both elements exhibit a strong tendency to polymorphism [36], which ensures that the pool of structures generated by MH will be large and diverse. Several studies in literature have already predicted conventional superconductivity with sizable $T_{c}$ in the BC system for selected compositions and structural motifs [18,19,37,38]; a series of pioneering works by Moussa and Cohen analyzed $T_{c}$ trends in selected templates, using the rigid-band (RB) approximation for doping and the rigid-muffin-tin approximation for the ep coupling [39-41]. Moreover, recent experimental claims exist of a record $T_{c}$ of $T_{c}=55 \mathrm{~K}$ (higher than in $\mathrm{MgB}_{2}$ ) in B-doped (amorphous) Q carbon, which is consistent with an ep mechanism [42-45].

In this paper, we exploit CSP methods to generate realistic metastable BC structures and systematically investigate their superconducting properties.

The $\mathrm{BC}$ phase diagram is extremely complex; hexagonal and tetrahedral motifs, characteristic of $\mathrm{C} s p^{2} / s p^{3}$ bonds, dominate the energy landscape up to $\sim 1 / 3: 2 / 3 \mathrm{C}$ :B compositions, while more complex motifs develop for higher $\mathrm{B}$ concentrations, due to an increasing role of electron-deficient boron [36]. To limit the scope of our analysis, we decided to focus on the single $50 \%-50 \%$ composition, where the physics and chemistry should still be dominated by carbon, but boron is in a sufficiently high concentration to ensure that many phases will exhibit a pronounced metallic behavior.

This paper is organized as follows. In Sec. II, we discuss the general features of the whole pool of 320 structures, their classification into different families, and their salient qualities. We also briefly describe how the 16 representative structures are selected for our subsequent superconductivity studies. In Sec. III, we discuss the trends in $T_{c}$ among different structures and how they are correlated with electronic structure quantities. In Sec. IV, we discuss in greater detail the electronic, vibrational, and superconducting properties of the structures. In Sec. V, we show that a simple analytical expression interpolates the $T_{c}$ of the representative structures and may be used as a predictor for superconductivity. Finally, in Sec. VI, we summarize the main conclusions of our paper. Appendix A contains plots of the electronic and phononic density of states (DOS) and Migdal-Eliashberg spectral functions for the 16 representative structures, while the methodology used for this paper is discussed in greater detail in Appendix B. In addition, the convex hull has also been provided in Appendix B.

\section{STRUCTURES : THERMODYNAMICS AND PROTOTYPES}

All structures considered in this paper have a 50\%-50\% $\mathrm{B} / \mathrm{C}$ stoichiometry and can be described with an eight-atom unit cell $\left(\mathrm{B}_{4} \mathrm{C}_{4}\right)$. This choice leaves out some interesting structural prototypes, such as nanotubes and fullerenes, but is a reasonable compromise between computational efficiency and structural flexibility.

Our initial MH runs produced around $\sim 630$ such structures. Through postrelaxation of this initial pool with tighter settings and removal of duplicates, we ended up with a final tally of 320 unique structures. The energy versus volume plot of these structures is shown in the upper panel of Fig. 1. The energy shown here is the formation energy, computed using the graphite structure for carbon and $\alpha$-rhombohedral- $\mathrm{B}_{12}$ for boron as references. The formation energy has been evaluated using Eq. (B1). All the BC structures are metastable with positive formation energies in the range of $0.1-1.0 \mathrm{eV} /$ atom. Although large, these values lie within the synthesizability threshold defined in Ref. [47] for carbon polymorphs. Also note that the formation energy may be sensibly reduced by choosing a different initial carbon structure for the synthesis which is closer to the target candidate.

The colors and symbols in Fig. 1 indicate the different families each structure belongs to, i.e., blue triangles for graphite $(G)$, inverted green triangles for slab (S), grey circles for diamond (D), and red circles for tubulane (T). The classification of the structures into different families was done by hand, based on the relative arrangement of tetrahedral/triangular motifs and on the fraction of $s p^{1}, s p^{2}$, and $s p^{3}$ bonds.

The combined variation of bonding fraction and the spatial arrangement of the tetrahedra and triangles formed by $s p^{3}$ or $s p^{2}$ bonds make some of the BC structures different from diamond or graphite. Their motifs are similar to those which have already been reported in pure carbon structures [48-54], and classified as interpenetrating graphene networks (IGN) [48] or carbon honeycombs (CHC) [54]. We have grouped these structures under the general keyword tubulanes. In addition, we have created a category slab to accommodate structures whose representative motifs have not been reported.

The characteristic features of the four different families are summarized below:

(i) Diamond [grey circles, Fig. 1(a)]: Diamond structures are characterized by dominant $s p^{3}$ bonding, which makes them occupy small volumes. Indicated by grey circles, the diamond structures are situated on the left side in the volume versus energy plot in Fig. 1.

(ii) Graphite [blue triangle, Fig. 1(b)]: Graphite structures are characterized by atomically thin layers stacked on top of each other. The majority of atoms within one layer are bonded through $s p^{2}$ bonding. The layers interact weakly through van der Waals interaction. This makes them occupy large atomic volumes as indicated by the location of the blue triangles in the right half of Fig. 1.

(iii) Slab [inverted green triangles, Fig. 1(c)]: A slab structure is geometrically similar to a graphite structure. However, at variance with graphite, formed by equispaced single layers, a slab structure is formed by slabs of multiple atomic layers, separated by void. As shown in Fig. 1(c), each slab comprises four atomic layers. Despite having finite thickness, these slabs experience weak van-der Waals interaction between them with an interslab distance of $\sim 2.9 \AA$. The atoms in this kind of system can form a mixture of $s p^{2}-s p^{3}$ bonds and hence occupy a large range of volumes. This is clearly evident from the large spread of the inverted green triangles as in Fig. 1. 


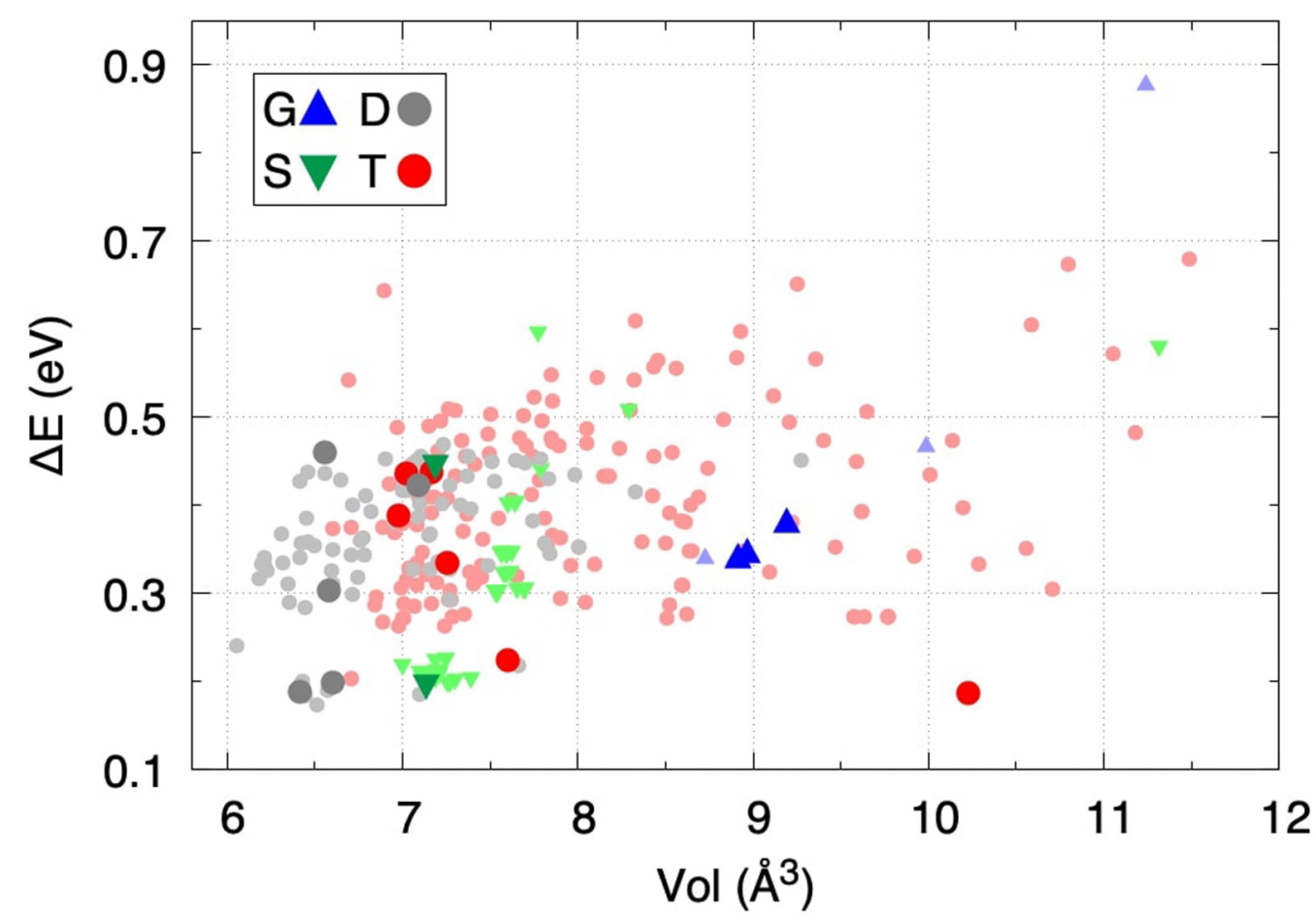

(a) Diamond

(b) Graphite

(c) Slab

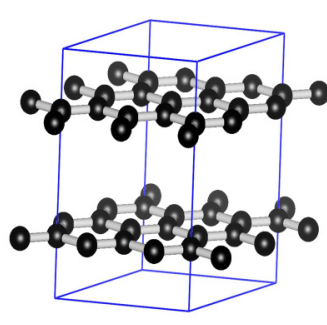

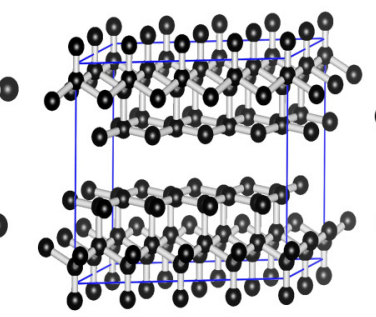

(d) Tubulane

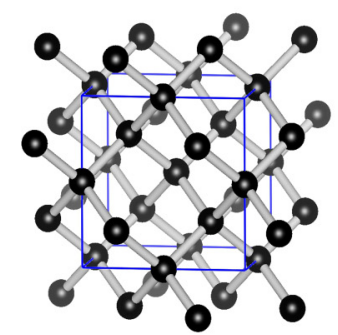

FIG. 1. The volumes versus formation energies of all predicted BC structures are shown in $\left(\AA^{3} /\right.$ atom) and (eV/atom), respectively. The formation energy is calculated with respect to the energy of graphite and $\alpha$-rhombohedral- $\mathrm{B}_{12}$. The colored symbols in the plot indicate the family of the structure, i.e., diamond (D) structures are represented by grey circles, graphite (G) by blue triangles, slab (S) by inverted green triangles, and tubulane (T) by red circles. Large dark-colored symbols indicate the 16 representative structures which we selected for further study, while the rest are shown by small light-colored symbols. Typical structures of the families diamond, graphite, slab, and tubulane are shown in (a)-(d), respectively; black spheres represent atoms and off-white cylinders bonds. Considering the full convex hull, the formation enthalpy may be uniformly shifted up or down, depending on the carbon precursor.

(iv) Tubulane [red circles, Fig. 1(d)]: The word tubulane, reported by Baughman and Galvao in 1993, refers to structures which display 3D networks of tubular structures [55]. These tubes can be of any shape, i.e., rhombohedral, hexagonal, circular, etc. The structures of the family IGN and $\mathrm{CHC}$ mentioned above fall in this category. As a typical example, Fig. 1(d) displays a tubulane with rhombohedral tubes. The constituent atoms in a tubulane can be connected via $s p^{1}, s p^{2}, s p^{3}$ bonds. With the possibility of having diverse mixture of bonds and tubes of different shapes, tubulanes can exhibit wide variability of atomic volumes, as shown by the wide distribution of the red circles in Fig. 1.

Note that a proper estimate of the formation enthalpy of BC structures should take into account not only the B and C end members, but also intermediate compositions. We thus evaluated the convex-hull of $\mathrm{BC}$, including the icosahedral structure with $\mathrm{B}_{13} \mathrm{C}_{2}$ composition, which is the lowest phase on the extended hull according to Ref. [36]. Taking the $\mathrm{B}_{13} \mathrm{C}_{2}$ phase into account, the formation enthalpies in Fig. 1 are shifted uniformly $66 \mathrm{meV}$ up. If, instead of the ground-state graphite $\mathrm{C}$, amorphous carbon is considered as a reference, the $\mathrm{BC}$ composition falls back on the hull; the actual formation enthalpies are then $470 \mathrm{meV}$ lower than in Fig. 1-see Appendix B, Fig. 21. Many of the BC phases considered in this paper may thus be synthesized, using the appropriate $\mathrm{C}$ precursor.

\section{SUPERCONDUCTING TRENDS OF REPRESENTATIVE STRUCTURES}

Superconductivity calculations are around two orders of magnitude more expensive than the total energy and structural relaxation runs used to construct our initial DB of structures. To narrow down our pool of potential superconductors, we first pruned out those structures, which have formation energies too high to be synthesizable, are dynamically unstable or 

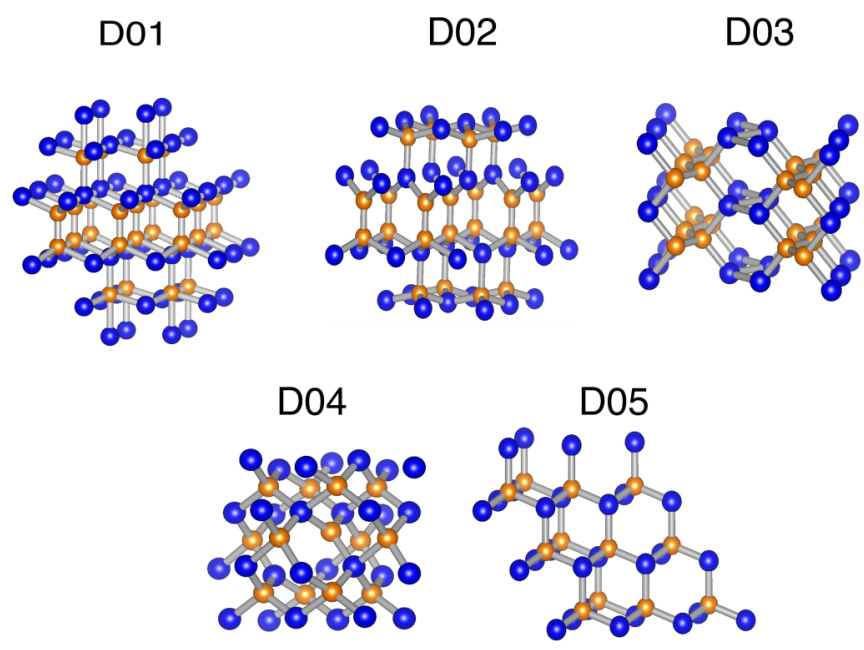

FIG. 2. Representative BC crystal structures in the diamond family. The $\mathrm{B}$ atoms are shown as blue spheres, $\mathrm{C}$ atoms as orange spheres and bonds in grey. The structures are marked by their ID.

exhibit poor metallic character and lack the stiff bonds, which are essential prerequisites for conventional HTS.

This was done by computing the value of electronic DOS at the Fermi level $\mathrm{N}\left(E_{F}\right)$ and the vibrational frequencies at the zone center $\left(\omega_{i}\right)$ for all structures in the DB. These quantities, together with the formation energy $\Delta \mathrm{E}$, were used to perform a preliminary screening, which left us with 116 potential candidates for HTS. As this number was still an order of magnitude too large to afford full $T_{c}$ calculations, we manually hand picked 16 representative candidates for accurate superconductivity calculations, shown as dark colored symbols in Fig. 1. In this second selection, we tried to choose structures with diverse structural motifs and arrangements of B-C bonds. A detailed description of the screening protocol and the criteria of selection can be found in Appendix B.

The 16 representative structures have been further relaxed with a Perdew-Wang-1992- Local Density Approximation (LDA) [56] functional before studying their geometric, electronic, vibrational, and superconducting properties. This second relaxation was needed because it is well known that structural and dynamical properties of layered (van der Waals systems) systems are poorly described within GGA, while LDA gives a reasonable account; the explicit inclusion of van der Waals corrections in density functional perturbation theory (DFPT) calculations of the $e p$ interaction is not yet implemented in any publicly available code.

Grouped by family, the structures are shown in Figs. 2-4 and their properties are summarized in Table I. In the following, the structures are represented by an alphanumeric ID of the form $\mathrm{A} X Y$, where A represents the family (diamond, graphite, tubulane, slab) and $X Y$ the energetic ranking. The CIF files of the 16 representative structures can be found in the Supplemental Material [57].

The first column in Table I lists the ID of the selected structures. General quantities describing the geometry are in the second (space group index), third (volume per atom), and last three columns, which indicate the presence $(\checkmark)$ or absence $(\boldsymbol{X})$ of bonds between C-C, B-B, and B-C, respectively [58]

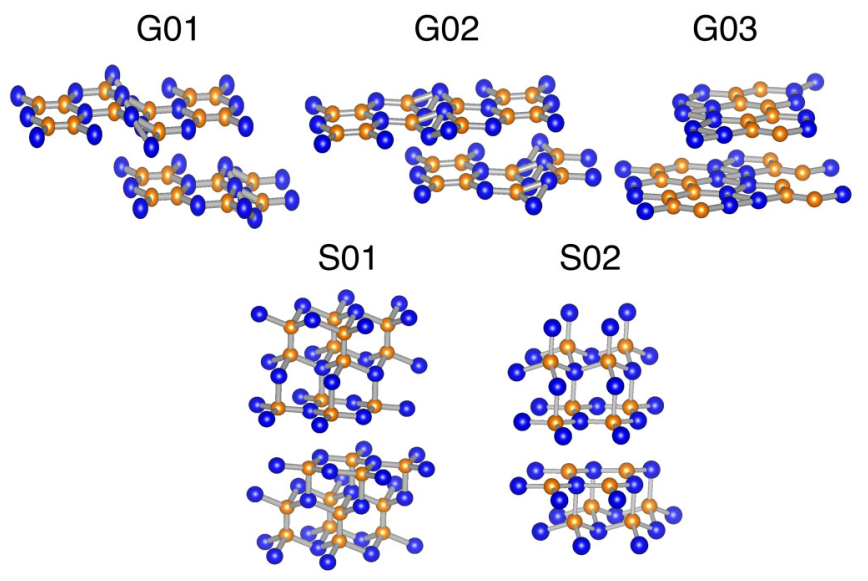

FIG. 3. Representative $\mathrm{BC}$ crystal structures in the graphite and slab family. The $\mathrm{B}$ atoms are shown as blue spheres, $\mathrm{C}$ atoms by orange spheres and bonds in grey. The structures are marked by their ID.

The threshold of distance for the presence of C-C, B-B, and $\mathrm{B}-\mathrm{C}$ bonds are $\mathrm{d}_{\mathrm{CC}} \leqslant 1.5 \AA, \mathrm{d}_{\mathrm{BB}} \leqslant 1.85 \AA$, and $\mathrm{d}_{\mathrm{BC}} \leqslant 1.65$ $\AA$, respectively. The formation energy $(\Delta E)$ and the electronic DOS at the Fermi level $\mathrm{N}\left(E_{F}\right)$ are in the fourth and fifth columns, respectively. Note that, in contrast to Fig. 1, here the $\Delta E$ in each family is reported considering as reference the lowest-energy $\mathrm{C}$ structure within that family. For the $\mathrm{D}$ and $\mathrm{G}$ families, diamond and graphite were considered. For the $\mathrm{S}$ family, diamond was considered. For the $\mathrm{T}$ family, the structure $I G N-Z 33$ from Ref. [52] was considered, which is a member of the $I G N$ family and the lowest energy $(0.1 \mathrm{eV} /$ atom with respect to graphite) structure in the tubulane family. This structure has symmetric rhombohedral tubes where the four sides are made of three $\mathrm{C}$ chains arranged in zigzag fashion, hence the name Z33.

Column six and seven list the $\left(\omega_{\max }\right)(\mathrm{meV})$ and the average $\left(\omega_{\mathrm{avg}}\right)(\mathrm{meV}) \Gamma$-point vibrational frequency, evaluated on an eight-atom unit cell for all structures. The quantities

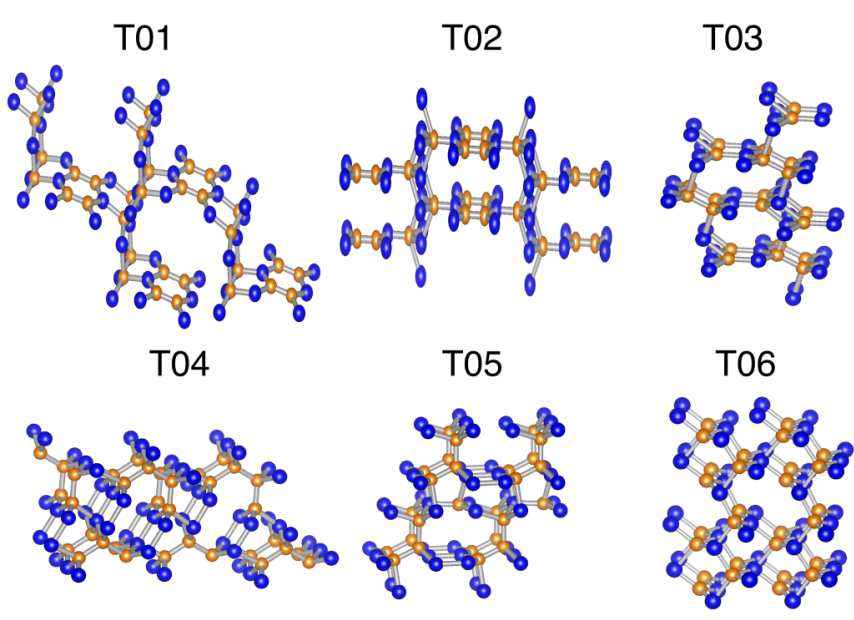

FIG. 4. Representative BC crystal structures in the tubulane family. The $\mathrm{B}$ atoms are shown as blue spheres, $\mathrm{C}$ atoms as orange spheres and bonds in grey. The structures are marked by their ID. 
TABLE I. Summary of calculated properties of representative BC structures belonging to different families, i.e., diamond (D), graphite $(\mathrm{G})$, slab (S), and tubulane (T). The structures are represented with an ID (first column), where the first letter represents the family and the last two integers, their energy ranking. The space group indexes of the structures are listed in the second column. The quantities volume $\left(\AA^{3}\right)$, $\operatorname{energy}(\Delta \mathrm{E}$ in $\mathrm{meV})$, and electronic density of states at the Fermi level $\mathrm{N}\left(E_{F}\right)$ (states/eV) are given per atom. For each family, the lowest-energy $\mathrm{C}$ structure and the e $\alpha$-rhombohedral- $\mathrm{B}_{12}$ are considered as references for computing the formation energy of the structures. Quantities $\omega_{\text {max }}$ (maximum frequency at the $\Gamma$ point), $\omega_{\text {avg }}$ (average of the optical vibrational frequencies at the $\Gamma$ point) calculated for an eight-atom unit cell and the logarithmic average phonon frequency $\omega_{\log }$ are in meV. The $e p$ coupling constant $\lambda$ is dimensionless and the $e p$ matrix element $\lambda / \mathrm{N}\left(E_{F}\right)$ is in (states/eV/atom $)^{-1}$. The superconducting critical temperature $T_{c}$ in $\mathrm{K}$ has been estimated using the McMillan-Allen-Dynes formula [46] with $\mu^{*}=0.10$. The last three columns lists the presence $(\checkmark)$ or absence $(\boldsymbol{X})$ of C-C, B-B, and B-C bonds, respectively.

\begin{tabular}{|c|c|c|c|c|c|c|c|c|c|c|c|c|c|}
\hline \multirow[t]{2}{*}{ ID } & \multirow{2}{*}{$\begin{array}{c}\text { Space } \\
\text { Gr. Ind. }\end{array}$} & \multirow{2}{*}{$\begin{array}{l}\text { Vol } \\
\left(\AA^{3}\right)\end{array}$} & \multirow{2}{*}{$\begin{array}{c}\Delta \mathrm{E} \\
(\mathrm{meV})\end{array}$} & \multirow{2}{*}{$\begin{array}{c}\mathrm{N}\left(E_{F}\right) \\
\text { (states/eV) }\end{array}$} & \multirow{2}{*}{$\begin{array}{l}\omega_{\max } \\
(\mathrm{meV})\end{array}$} & \multirow{2}{*}{$\begin{array}{c}\omega_{\text {avg }} \\
(\mathrm{meV})\end{array}$} & \multirow[t]{2}{*}{$\lambda$} & \multirow[t]{2}{*}{$\lambda / \mathrm{N}\left(E_{F}\right)$} & \multirow{2}{*}{$\begin{array}{c}\omega_{\log } \\
(\mathrm{meV})\end{array}$} & \multirow{2}{*}{$\begin{array}{l}T_{c} \\
(\mathrm{~K})\end{array}$} & \multicolumn{3}{|c|}{ Bond Type } \\
\hline & & & & & & & & & & & $\mathrm{CC}$ & $\mathrm{BB}$ & $\mathrm{BC}$ \\
\hline \multicolumn{14}{|c|}{ Diamond } \\
\hline D01 & 164 & 6.30 & 170 & 0.15 & 160 & 79 & 0.6 & 4.2 & 72 & 21 & $\checkmark$ & $x$ & $\checkmark$ \\
\hline D02 & 008 & 6.47 & 190 & 0.12 & 162 & 84 & 0.5 & 4.3 & 62 & 10 & $\checkmark$ & $x$ & $\checkmark$ \\
\hline D03 & 051 & 6.46 & 270 & 0.16 & 152 & 92 & 0.8 & 4.9 & 72 & 35 & $\checkmark$ & $\checkmark$ & $\checkmark$ \\
\hline D04 & 160 & 6.92 & 420 & 0.21 & 128 & 92 & 0.8 & 3.9 & 52 & 30 & $x$ & $x$ & $\checkmark$ \\
\hline D05 & 216 & 6.56 & 440 & 0.36 & 106 & 71 & 2.3 & 6.3 & 41 & 75 & $x$ & $x$ & $\checkmark$ \\
\hline \multicolumn{14}{|c|}{ Graphite } \\
\hline G01 & 012 & 8.57 & 360 & 0.09 & 186 & 95 & 0.4 & 4.1 & 42 & 2 & $\checkmark$ & $\checkmark$ & $\checkmark$ \\
\hline G02 & 012 & 8.63 & 360 & 0.11 & 186 & 95 & 0.4 & 3.4 & 39 & 1 & $\checkmark$ & $\checkmark$ & $\checkmark$ \\
\hline G03 & 002 & 8.91 & 390 & 0.08 & 198 & 104 & 0.4 & 4.3 & 32 & 1 & $\checkmark$ & $\checkmark$ & $\checkmark$ \\
\hline \multicolumn{14}{|l|}{ Slab } \\
\hline S01 & 164 & 6.96 & 190 & 0.16 & 161 & 76 & 0.6 & 4.1 & 79 & 25 & $\checkmark$ & $x$ & $\checkmark$ \\
\hline S02 & 156 & 7.07 & 440 & 0.21 & 138 & 72 & 1.1 & 5.3 & 57 & 53 & $x$ & $x$ & $\checkmark$ \\
\hline \multicolumn{14}{|c|}{ Tubulane } \\
\hline T01 & 044 & 10.02 & 150 & 0.14 & 184 & 98 & 0.3 & 1.9 & 44 & 0 & $\checkmark$ & $x$ & $\checkmark$ \\
\hline T02 & 071 & 7.47 & 180 & 0.14 & 156 & 95 & 0.6 & 3.9 & 42 & 9 & $\checkmark$ & $x$ & $\checkmark$ \\
\hline T03 & 012 & 7.12 & 260 & 0.25 & 163 & 88 & 0.7 & 2.8 & 58 & 24 & $\checkmark$ & $x$ & $\checkmark$ \\
\hline T04 & 044 & 6.83 & 310 & 0.16 & 149 & 90 & 0.6 & 3.9 & 51 & 15 & $\checkmark$ & $\checkmark$ & $\checkmark$ \\
\hline T05 & 001 & 6.85 & 360 & 0.21 & 149 & 85 & 1.2 & 5.4 & 37 & 37 & $\checkmark$ & $\checkmark$ & $\checkmark$ \\
\hline T06 & 006 & 7.00 & 380 & 0.22 & 138 & 84 & 0.9 & 4.1 & 60 & 42 & $x$ & $x$ & $\checkmark$ \\
\hline
\end{tabular}

$\Delta E, \mathrm{~N}\left(E_{F}\right)$, and $\omega_{\text {avg }}$ have been used for a prescreening of structures as discussed in Appendix B.

Quantities associated with superconducting properties listed in Table I are the $e p$ coupling constant $\lambda$, the approximate effective $e p$ matrix element $\lambda / \mathrm{N}\left(E_{F}\right)$, the logarithmic average phonon frequency $\omega_{\log }(\mathrm{meV})$, and the superconducting critical temperature $T_{c}(\mathrm{~K})$ estimated with the McMillanAllen-Dynes formula [46],

$$
T_{c}=\frac{\omega_{\log }}{1.2} \exp \left[-\frac{1.04(1+\lambda)}{\lambda-\mu^{*}(1+0.62 \lambda)}\right]
$$

with a standard value $\mu^{*}=0.10$ for the Coulomb pseudopotential.

\section{A. Structural properties}

The structures listed in Table I are shown in Figs. 2-4. All diamond structures, which consist of a mixture of $s p^{2}$ and $s p^{3}$ bonds, contain B-C bonds. Structures D01 and D02 also contain $\mathrm{C}-\mathrm{C}$ bonds. The $\mathrm{C}-\mathrm{C}$ bond is shared by two opposite-facing tetrahedra, while the other three bonds of the tetrahedron are B-C bonds. Structure D03 is the only structure in the D family which contains B-B bonds. The structure consists of zigzag chains of $\mathrm{C}$ and $\mathrm{B}$ ordered in a particular fashion to form $s p^{3}$ bonds. Both D04 and D05 only involve $\mathrm{B}-\mathrm{C}$ bonds. The major difference between the two is that the structure D04 encompasses a mixture of $s p^{2}$ and $s p^{3}$ bonds, whereas D05 only contains $s p^{3}$ bonds.

The layers of the graphitic structures G01 and G02 are the same and the two structures only differ in the relative arrangement of the layers. Unlike the layers of pure $\mathrm{C}$ graphite, which are flat, these layers have a staircase shape and consist of hexagons formed by C-C and B-C bonds. The B atoms which form the edge of the staircase have coordination number $\sim 4-5$, and hence form bonds which cannot be classified as purely $s p^{2}$ or $s p^{3}$. The structure G03 contains flat atomic layers like graphite, in which arm-chair chains of $\mathrm{C}$ atoms are connected to ordered chains of multibonded $\mathrm{B}$ atoms. These two chains of $\mathrm{C}$ and $\mathrm{B}$ form hexagons and pentagons along with the clustering of B atoms. Like every graphitic structure, there is a large interlayer distance.

The two slab structures S01 and S02 have identical structural templates; the only difference is that the $\mathrm{B}$ and $\mathrm{C}$ atoms in S02 are arranged such that they only have B-C bonds whereas S01 also has C-C bonds along with B-C bonds. Each slab layer consists of four atomic layers; the two inner layers are bonded through $s p^{3}$ bonds whereas the two outer layers are bonded through $s p^{3}$ bonds with the inner layer, while the remaining bonds are $s p^{2}$ like. The two outer layers contain hexagons.

The representative structures of the tubulane family all encompass 3D tubes of different shapes and sizes, with different fractions of $s p^{2}-s p^{3}$ bonds. Tubes with large diameter occupy 
larger volumes, as seen in T01 and T02. Like every other structural templates, also in the tubulane family all members contain B-C bonds; in addition, all members except T06 contain $\mathrm{C}-\mathrm{C}$ bonds. The $\mathrm{C}$ atoms in $\mathrm{C}-\mathrm{C}$ bonds are part of an $s p^{2}$ geometry in structure $\mathrm{T} 01$ and $\mathrm{T} 02$. In the remaining cases, they are in an $s p^{3}$ geometry. Structures T04 and T05 contain B-B bonds which are part of buckled hexagons, arranged in a $s p^{2}-s p^{3}$ geometry. Structure T06 only contains B-C bonds, which are in a $s p^{3}$ geometry.

\section{B. Trends in $T_{c}$}

The 16 structures listed in Table I represent a diverse sample of possible structural motifs and properties. Before analyzing their electronic structure in detail, some general trends across and within families can already be discussed on the basis of the data in Table I.

In general, three observations are in place:

(1) In all families except graphite, we found structures with rather high values of the DOS at the Fermi level $\mathrm{N}\left(E_{F}\right)$, and moderate to high $T_{c}$ 's. We also observe that these structures with higher DOS and $T_{c}$ tend to have quite high formation energies $\Delta E$, of the order of $200-400 \mathrm{meV}$, which is close to the synthetizability threshold [47]. The structure with the highest $\mathrm{N}\left(E_{F}\right)$ is D05, which is a diamond structure with only B-C bonds in a perfect tetrahedral geometry. Other structures with high $\mathrm{N}\left(E_{F}\right)$ are D04, S02, T05, and T06, which all exhibit $T_{c}$ 's exceeding $30 \mathrm{~K}$. All graphitic structures obtained from $\mathrm{MH}$ runs have rather small values of $\mathrm{N}\left(E_{F}\right)$, and negligible $T_{c}$ 's. We thus tried to construct graphitic structures with high $\mathrm{N}\left(E_{F}\right)$ manually, through different homogeneous replacements of B in $\mathrm{C}$ graphite in an eight-atom cell. However, we found that any arrangement of B atoms in C graphite induces buckling, and that these buckled structures are either dynamically stable nonmetals or dynamically unstable metals. This observation confirms what has been observed in studies of $\mathrm{B} / \mathrm{N}$ doping of single graphene sheets by Zhou et al. and Mann et al. $[59,60]$.

(2) A second quantity exhibiting a remarkable correlation with the $T_{c}$ is the value of the highest vibrational frequency at the $\Gamma$ point $\left(\omega_{\max }\right)$ and, in particular, its reduction (softening) with respect to the same quantity in a reference structure of pure carbon. In general, the softening is more pronounced for structures with higher $\mathrm{N}\left(E_{F}\right)$ and $\lambda$. Almost all diamond structures exhibit a remarkable softening of the highest vibrational frequencies $\left(\omega_{\max }\right)$, with respect to that of pure diamond (164 meV) [61]. The softening is the highest for D05, where $\omega_{\max }$ is reduced by a factor of 0.65 compared to the reference value. On the contrary, graphite structures exhibit only a small softening, as compared to the the reference value for C graphite $(195 \mathrm{meV})$. Tubulane structures also exhibit a strong softening of $\omega_{\max }$, compared to the reference tubulane structure (ING-Z33 $200 \mathrm{meV}$ ). It is hard to give a quantitative estimate of this effect for slab structures, because no dynamically stable reference structure exists, but the reference value should lie somewhere between $s p^{3}$ diamond and $s p^{2}$ graphite, and both S01 and S02 exhibit a remarkable softening with respect to this value.

(3) A third, more general correlation can be found across the whole DB between $T_{c}$ and the types of bonds (B-B, B-C or $\mathrm{C}-\mathrm{C}$ ) present. In particular, structures which contain B-C bonds only have the highest $T_{c}$ within each family. Structures with and without $\mathrm{C}-\mathrm{C}$ and B-B bonds along-with B-C bonds may or may not be superconductors. A close look at Figs. 2-4 show that in structures containing B-B bonds, the B atoms are not part of $s p^{2}-s p^{3}$ bonds, but form multiple bonds. This leads to a sizable deformation of the structure, which reduces the symmetry and causes a sensible reduction of $N\left(E_{F}\right)$, and hence $T_{c}$. For example, B atoms in G01, G02, and G03 form four to five bonds with both B and C. On the other hand, the role of C-C bonds in determining $T_{c}$ is much less clear. Finally, it is interesting to note that, though both D04 and D05 only contain B-C bonds, they have different $T_{c}$ 's. This difference can be associated to the fact that D05 only contains $s p^{3}$ bonds, whereas D04 contains a mixture of $s p^{2}-s p^{3}$ bonds, and also in this case the symmetry lowering leads to a $T_{c}$ suppression.

\section{ELECTRONIC STRUCTURE}

In this section, we present a detailed comparison of the electronic structure of the representative structures to gain a microscopic insight of their superconducting properties, discussed only in general terms so far.

The electronic DOS, phonon and electron-phonon spectra (Eliashberg functions) for our 16 representative structures, divided by families, are reported in Appendix B, Figs. 9-20; in the electronic (Figs. 9-12) and phonon (Figs. 13-16) DOS plots, we report in red and blue the partial carbon and boron contributions as well as the total DOS in black. The top panels of all figures show reference spectra, calculated for a pure carbon structure.

The electronic DOS plots show an almost perfect hybridization between $\mathrm{B}$ and $\mathrm{C}$ states in all structures, with the two partial DOS closely following each other. In addition, the variation of the spectral distribution of the electronic states in different $\mathrm{BC}$ structures, compared to the reference pure carbon structures, is a good indicator of the changes in electronic structure due to rearrangement of bonds. In this respect, it is quite interesting to compare the behavior of structures in the diamond and graphite families, where it is straightforward to define a reference template for the pure structure. In both cases, in a simple RB model, the Fermi level, shown by the dashed line in the upper panels of Figs. 9-10, would fall into a $\sigma(2 \mathrm{D}$ or 3D) band. In this case, one would predict a sizable $e p$ coupling, as $\sigma$ bonds are extremely stiff and sensitive to lattice distortions. [18]

However, in most real structures, a substantial rearrangement of bonds and electronic states invalidates this simple line of reasoning, based on the the RB approximation. In the diamond family, a substantial shift of spectral weight away from the Fermi level occurs, which is more pronounced for low-energy structures, where it produces a substantial lowering of the DOS at the Fermi; the shift is absent in D05, which can almost perfectly be described by the RB approximation. In the graphite family, all structures generated for $50 \%$ BC composition are either dynamically unstable, or weakly metallic, due to a major rearrangement of bonds. G01, G02, and G03 all contain B-B and C-C dimers, and/or buckled planes, and exhibit an extremely small $N\left(E_{F}\right)$.

For slabs and tubulanes, due to the large variety of moieties and motifs, it is less straightforward to define a reference 
structure. We chose T06 and S02 as a structural template for the $\mathrm{C}$ reference structure for tubulane and slab, respectively. Also in these cases, a pronounced shift of spectral weight away from the Fermi level is observed, which is reduced for higher-energy structures. The DOS of low-energy tubulanes, which are more open, resemble quite closely those of graphite structures, while high-energy ones tend to mimic those of diamond. The same tendency can be observed in slab structures.

Phonon DOSare shown in Figs. 13-16, again with the same color code and definition of reference structures. As observed for electronic DOS, due to the similar B and C mass, the spectra have in general a fairly mixed character. However, the phonon DOS of structures which contain B-B or C-C bonds tend to exhibit sharp peaks of pure $\mathrm{B}$ or $\mathrm{C}$ character, corresponding to localized vibrations. Many of these peaks are found at high energies. In addition, a progressive reduction of the highest phonon frequency with increasing formation energy is also evident in all families. The effect is particularly spectacular in DO5, where the reduction of the highest frequency is $\sim 35 \%$.

Fgures 17-20 show for each family the Migdal-Eliashberg $e p$ spectral function [62],

$$
\alpha^{2} F(\omega)=\frac{1}{N\left(E_{F}\right)} \sum_{\mathbf{k q}, v}\left|g_{\mathbf{k}, \mathbf{k}+\mathbf{q}, v}\right|^{2} \delta\left(\epsilon_{\mathbf{k}}\right) \delta\left(\epsilon_{\mathbf{k}+\mathbf{q}}\right) \delta\left(\omega-\omega_{\mathbf{q}, v}\right),
$$

where $\mathrm{N}\left(E_{F}\right)$ is the electronic DOS at the Fermi level and the two $\delta$ functions restrict the sum to electronic states at the Fermi level with momenta $\mathbf{k}$ and $\mathbf{k}+\mathbf{q}$. The $\omega_{\mathbf{q}, v}$ is the vibrational frequency of mode $v$ and wave vector $\mathbf{q}$ and $g_{\mathbf{k}, \mathbf{k}+\mathbf{q}, v}$ is the corresponding electron-phonon matrix element. On the same plots, with orange dashed lines we show the frequency-dependent $e p$ constant $\lambda(\omega)$ and report the average phonon frequency $\omega_{\log }$, given by

$$
\begin{gathered}
\lambda(\omega)=2 \int_{0}^{\omega} \frac{\alpha^{2} F\left(\omega^{\prime}\right)}{\omega^{\prime}} d \omega^{\prime}, \\
\omega_{\log }=\exp \left[\frac{2}{\lambda} \int_{0}^{\infty} \alpha^{2} F(\omega) \frac{\ln (\omega)}{\omega} d \omega\right],
\end{gathered}
$$

which measure, respectively, the average energy of the phonons which couple mostly to electrons, and of the intensity of the ep coupling.

In most compounds, the Eliashberg function is almost proportional to the phonon DOS, reflecting a uniform spread of the $e p$ coupling on the phonon spectrum. A notable exception is the slab structure $\mathrm{S} 02$, where there is a substantial enhancement of coupling to phonons in the low-energy region. While the values of $\omega_{\log }$ are quite spread out, without any clear trend for low- or high-energy structures, the values of the total ep coupling constant $\lambda$, obtained from Eq. (3) with $\omega=\infty$, tend to be larger for higher-energy structures, and range from 0.4 in graphite structures $\mathrm{G} 01, \mathrm{G} 02$, and $\mathrm{G} 03$ to 2.3 in diamond D05.

The main factor behind the large variation in $\lambda$ amongst structures is the variation of the electronic DOS at the Fermi level $\mathrm{N}\left(E_{F}\right)$. This can be appreciated recalling that $\lambda$ can be rewritten using the so-called Hopfield expression [63],

$$
\lambda=\frac{N\left(E_{F}\right) I^{2}}{M \tilde{\omega}^{2}},
$$

where $I^{2}$ is the $e p$ coupling matrix element averaged over Fermi surface, $M$ is the average atomic mass, and $\tilde{\omega}^{2}$ is the square of an average vibrational frequency. As reported in Table I, $V=\frac{\lambda}{N\left(E_{F}\right)}$ is $\simeq 4.0$ in most structures considered in this paper. The only notable exception is the diamond structure D05, where this ratio is 50\% larger than in all other structures, reflecting a qualitative difference in bonding with respect to all other structures.

In summary, the analysis of the electronic structure shows that most structural templates exhibit a similar tendency to superconductivity: the $e p$ coupling is spread out over several phonon modes, and the value of the $e p$ coupling constant $\lambda$, and hence $T_{c}$, is mostly determined by the value of $\mathrm{N}\left(E_{F}\right)$, since the ratio $V=\lambda / N\left(E_{F}\right)$, is essentially constant across and within families. In most low-energy structures $T_{c}$ is suppressed by the formation of B-B and C-C bonds, which shifts electronic spectral weight away from the Fermi level, lowering the band energy, but also $\mathrm{N}\left(E_{F}\right)$.

The presence of B-B and C-C bonds is also visible in the phonon spectra, where it leads to the formation of sharp peaks at high energies.

The diamond structure D05, where, due to the alternating arrangement of B-C atoms, the original symmetry of pure diamond is retained, and electronic states at the Fermi level have a pure $\sigma\left(s p^{3}\right)$ character, is a clear outlier of the DB. Here, the DOS follows a perfect RB behavior compared to pure diamond, while the phonon spectrum is strongly renormalized, due to coupling between bond-stretching phonons and $\sigma$ states. As a result, $V=\lambda / N\left(E_{F}\right)$ is around $50 \%$ larger than in all other representative structures, and the predicted superconducting $T_{c}$ is also exceptional $(79 \mathrm{~K})$, in line with the highest values calculated in Ref. [39].

These observations imply that general arguments based on the RB analysis of fixed structural templates must be taken with care in $\mathrm{BC}$ [41] because structural distortions and bond rearrangements can have a dramatic effect on $T_{c}$.

\section{A SIMPLE EXPRESSION FOR $T_{c}$}

The values of $T_{c}, \lambda$ and $\omega_{\log }$ for all 16 representative structures, collected in Table I, were computed using the McMillan-Allen-Dynes formula, Eq. (1), which requires a full calculation of the electron-phonon (Eliashberg) spectral function, Eq. (2).

For an eight-atom unit cell with no symmetry, a calculation of $\alpha^{2} F(\omega)$ with a reasonably dense sampling of reciprocal space for electronic and phononic momenta requires around $3000 \mathrm{CPU}$ hours on a computer cluster. This type of calculation is clearly unfeasible for large-scale high-throughput material screening, which was our primary motivation to preselect only a few representative structures from our initial pool.

With the $T_{c}$ data at hand, it is interesting to see whether any trends in $T_{c}$ could have been foreseen on the basis of the simple electronic structure quantities that we had used to prescreen our structural DB, which require a much less intense computational effort.

The three upper panels of Fig. 5 show that $T_{c}$ exhibits an almost linear correlation with $\mathrm{N}\left(E_{F}\right)$, and an inverse correlation with both $\omega_{\max }$ and $\omega_{\text {avg. }}$. Although the two vibrational 

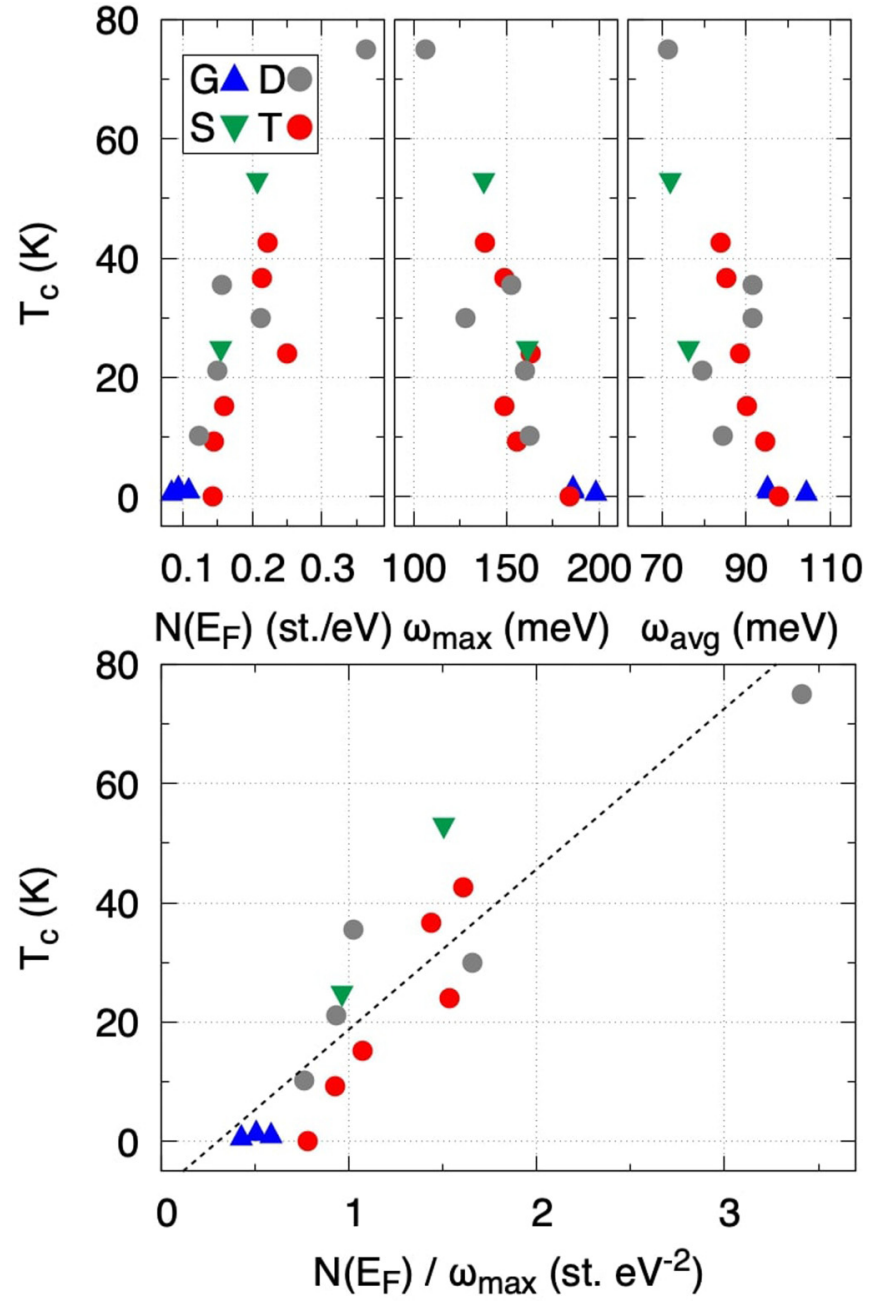

FIG. 5. Top panel: Superconducting critical temperature $T_{c}(\mathrm{~K})$ of the 16 representative $\mathrm{BC}$ structures as a function of (left) electronic DOS at the Fermi level $\mathrm{N}\left(E_{F}\right)$ (states/eV/atom) left, (middle) maximum vibrational frequency at the $\Gamma$-point $\omega_{\max }(\mathrm{meV})$; (right) average optical vibrational frequency at the $\Gamma$-point $\omega_{\max }(\mathrm{meV})$. Bottom panel: The $T_{c}$ 's of the 16 representative $\mathrm{BC}$ structures are plotted as a function of $\mathrm{N}\left(E_{F}\right) / \omega_{\max }$ in (states $/ \mathrm{eV}^{2} /$ atom). The color and the symbols in the plot indicate the family each structure belongs to. The dotted line represents an approximate linear fit to the $T_{c}$; data-Eq. (6).

descriptors are approximately equivalent, $\omega_{\max }$ is monotonous, while $\omega_{\text {avg }}$ incorrectly classifies the two slab structures and a few diamond ones. The lower panel of Fig. 5 shows that the calculated $T_{c}$ 's when plotted as a function of $\frac{N\left(E_{F}\right)}{\omega_{\max }}$ closely follow a linear behavior:

$$
T_{c}=26.9 \text { Kstates }^{-1} \mathrm{eV}^{2} \cdot\left[\frac{N\left(E_{F}\right)}{\omega_{\max }}-0.3\right]
$$

extremely simple, this formula seems to interpolate nicely the $T_{c}$ from different templates and has a transparent physical interpretation.

That $T_{c}$ should positively correlate with $\mathrm{N}\left(E_{F}\right)$ can be easily understood from the Hopfield's expression for $\lambda-$ Eq. (5). On the other hand, the correlation of $T_{c}$ with $\omega_{\max }$ is less straightforward to understand. One aspect is probably

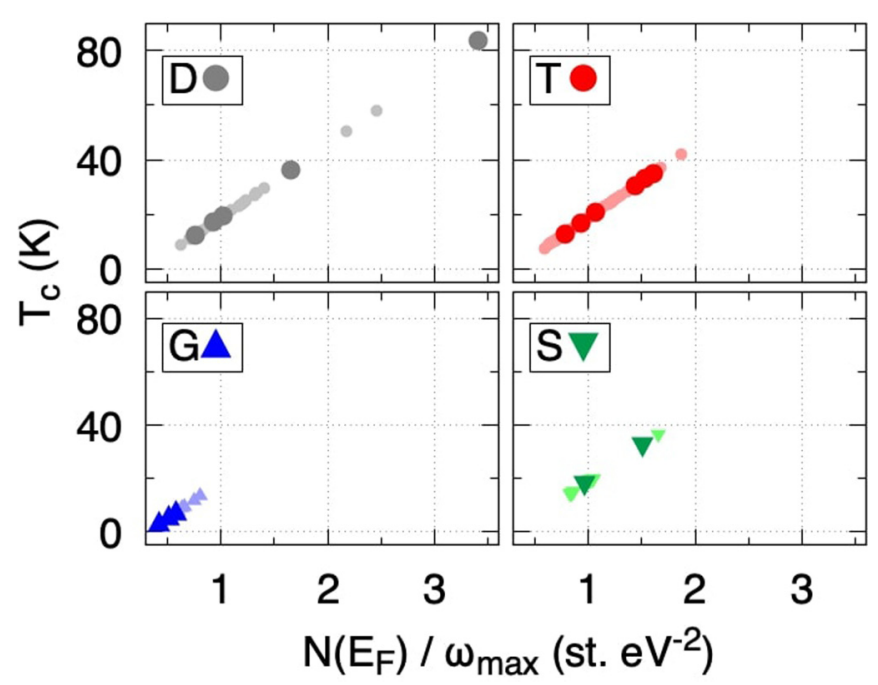

FIG. 6. The four panels show the model $T_{c}$ [Eq. (6)] with respect to $\mathrm{N}\left(E_{F}\right) / \omega_{\max }$ in (states $/ \mathrm{eV}^{2} /$ atom) for all the structures separated by families. Here, $\mathrm{N}\left(E_{F}\right)$ is the DOS at the Fermi level and $\omega_{\max }$ is the maximum vibrational frequency at the $\Gamma$ point. The top left panel is for diamond (grey circles), top right for tubulane (red circles), bottom left for graphite (blue triangles), and bottom right for slab (inverted green triangles). The selected structures are shown by big dark colored symbols. The rest are shown by small light colored symbols.

phonon softening: In an interacting system of phonons and electrons, the same coupling which leads to superconductivity also leads to the renormalization of phonon frequencies, with respect to a bare, noninteracting value. In a simple model where a single phonon mode with frequency $\omega$ couples to a single electronic band, $\omega$ is reduced with respect to its bare value $\Omega$ as $\omega^{2}=\Omega^{2}(1-2 \lambda)$.

However, while the model of a single phonon mode may be safely applied to hole-doped diamond and graphite, where

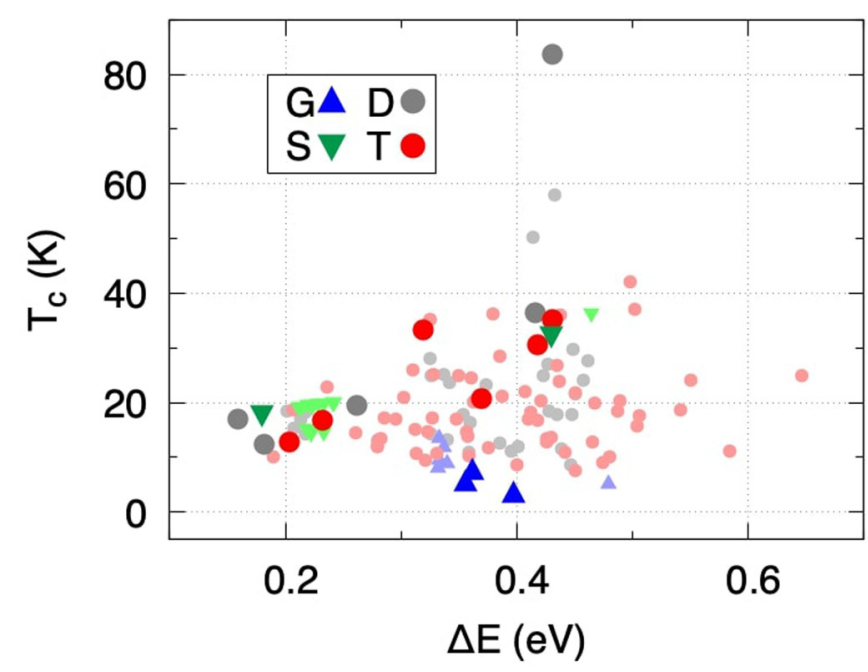

FIG. 7. Superconducting critical temperature $T_{c}(\mathrm{~K})$ of all the metallic $\mathrm{BC}$ structures as a function of formation energy in (eV/atom). The color and the symbols in the plot indicate the family each structure belongs to. 


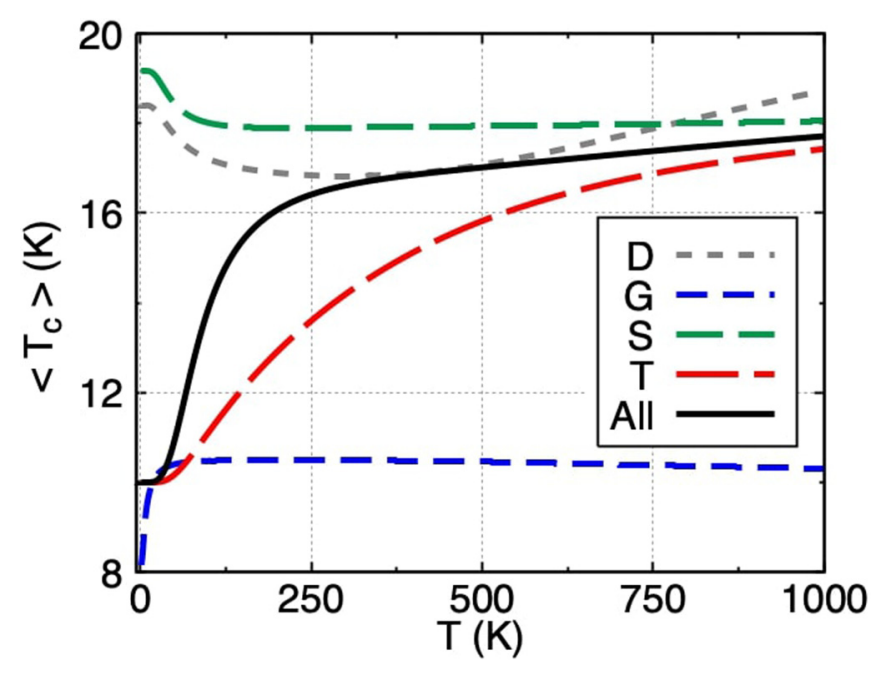

FIG. 8. The Boltzmann-averaged superconducting critical temperature $\left\langle T_{c}\right\rangle(\mathrm{K})$ for each prototype and all metallic BC structures as a function of temperature $(\mathrm{K})$. The $\left\langle T_{c}\right\rangle$ for each prototype was evaluated by weighing and normalizing the model $T_{c}$ obtained by using Eq. (6), with the Boltzmann factor based on their relative energies. The color of the lines indicate the family. The $\left\langle T_{c}\right\rangle$ for all the structures is shown by the black line.

superconductivity is dominated by the zone-center bondstretching optical phonon and $\sigma$ holes [18], its applicability to structures where the RB model does not hold due to major structural rearrangements and the coupling is spread out over several phonon modes and electronic states is questionable. It is in fact possible that $\omega_{\max }$ accidentally encodes both the presence of high-energy $\mathrm{B}-\mathrm{B}$ or $\mathrm{C}-\mathrm{C}$ phonon modes, due to the formation of $\mathrm{B}-\mathrm{B}$ and $\mathrm{C}-\mathrm{C}$ bonds, which are disruptive for HTCS, and the actual phonon softening of a large part of the phonon spectrum in systems where the coupling is strong. More tests are needed to check the general validity of this trend, even for a relatively specialized case as BC. This goes well beyond the aim of the present paper.

However, we can use our simple predictor for $T_{c}$ to estimate the tendency of BC structures to HTCS across the whole energy landscape. In Fig. 6, the model $T_{c}$ from Eq. (6), as a function of $\mathrm{N}\left(E_{F}\right) / \omega_{\max }$, is shown in four different panels for all metallic structures in the original DB, grouped by family. Around $60 \%$ of the predicted $T_{c}$ lie in the $1020 \mathrm{~K}$ range, $25 \%$ between 20 and $30 \mathrm{~K}$, and $\sim 10 \%$ are above $30 \mathrm{~K}$. These high- $T_{c}$ structures belong mostly to the tubulane and diamond families, a few to the slab family, whereas all graphite structures are predicted to exhibit $T_{c}$ 's below $20 \mathrm{~K}$. Note that also in this plot the structure D05, i.e., the data point with the highest $T_{c}$ in Fig. 6, is a complete outlier, and most likely its $T_{c}$ of $79 \mathrm{~K}$ is an upper bound for the BC system at 50\%-50\% composition.

Equation (6) can also be used to obtain an estimate of the formation energy required to obtain structures for a specific range of $T_{c}$. In Fig. 7, the predicted $T_{c}$ for all metallic BC structures is plotted as a function of formation energy; the meaning of colors and symbols is the same as in Fig. 1. We observe that, in general, the lowest-energy structures have $T_{c}$ 's below $20 \mathrm{~K}$. A large cluster of structures is also found, with $\Delta E$ between 300 and $400 \mathrm{meV}$, and $T_{c}$ 's exceeding $30 \mathrm{~K}$.
We can use the distribution of predicted $T_{c}$ 's to estimate which $T_{c}$ would result from a hypothetical experiment, in which a given carbon precursor would be heated together with boron and rapidly quenched down.

Assuming a simple percolation model, an average $\left\langle T_{c}\right\rangle(\mathrm{K})$ as a function of temperature $T(\mathrm{~K})$ can be estimated for each prototype as

$$
\left\langle T_{c}\right\rangle=\sum_{i} T_{c, i} \exp \left(-\frac{\Delta E_{i}}{k_{B} T}\right),
$$

where $T_{c, i}$ are obtained from Eq. (6) and the formation energy $\Delta E_{i}$ and $\mathrm{k}_{B}$ is the Boltzmann constant. The plot is shown in Fig. 8 where the different colors of the line indicate corresponding prototype. With increasing temperature $T$, the average $\left\langle T_{c}\right\rangle$ of $\mathrm{G}, \mathrm{D}$, and $\mathrm{S}$ families seems to saturate quite early at $100-200 \mathrm{~K}$ whereas the $T$ family seems to gradually saturate with increasing temperature. The highest percolation $\left\langle T_{c}\right\rangle$ is that of the $\mathrm{S}$ family at about $18 \mathrm{~K}$, closely followed by the $\mathrm{D}$ and $\mathrm{T}$ families. In brief, the $\mathrm{S}, \mathrm{D}$, and $\mathrm{T}$ family have similar $\left\langle T_{c}\right\rangle$, whereas for $\mathrm{G}$, the $\left\langle T_{c}\right\rangle$ is very small, $10 \mathrm{~K}$.

\section{CONCLUSIONS}

In this paper, we probed the possibility of realizing HTCS in the $\mathrm{BC}$ system, using an $a b$ initio screening approach. First, we generated a large (320) DB of metastable BC structures, with 50\%-50\% boron/carbon composition and eightatom unit cells, and showed that these can be grouped into four main families of characteristic motifs for pure carbon: diamond, graphite, slab, and tubulane. From a first highthroughput screening based on the values of the electronic DOS at the Fermi level, zone-center vibrational frequencies, and formation energies, we estimated that around half of the generated structures are promising HCTS. From these, we selected 16 representative structures, spanning a variety of motifs and structural templates, for which we performed full electron-phonon calculations. We identified several general trends amongst them: (i) In all families, except graphite, we could find superconductors with $T_{c}$ 's $\simeq 40 \mathrm{~K}$, comparable to the best-known ambient-pressure superconductors; (ii) within one family, the value of $T_{c}$ is essentially determined by $\mathrm{N}\left(E_{F}\right)$; (iii) $T_{c}$ correlates inversely with the highest phonon frequecty at the zone center, $\omega_{\max }$; and (iv) a geometric analysis of the selected structures shows that the highest $T_{c}$ 's within a given family is usually found in structures where the fraction of B-C bonds is dominant with respect to other types of bonds, and particularly if these have $s p^{3}$ character. Structures where bonds centered around $\mathrm{B}$ atoms are neither $s p^{2}$ nor $s p^{3}$ tend to exhibit a low $T_{c}$, because the clustering of atoms around B tends to reduce the symmetry, depress the value of $\mathrm{N}\left(E_{F}\right)$, and hence $T_{c}$. A similar behavior has been observed in amorphous Q carbon [45].

The empirical observations (i)-(iii) can be distilled into a single analytical formula for $T_{c}$, which can be used as a predictor for HTCS. On the basis of this formula, we estimate that around $\sim 10 \%$ structures have $T_{c}$ larger than $30 \mathrm{~K}$, which makes them interesting candidates for HTCS. Most of these structures have formation energies between 300 and $400 \mathrm{meV}$ and may be synthesized using an appropriate carbon precur- 
sor. The diamond structure D05, which only has B-C bonds in $s p^{3}$ tetrahedral arrangement, sets an upper limit for $T_{c} \sim 80 \mathrm{~K}$ for the BC system at $50 \%-50 \%$ composition. Given that $T_{c}$ is so strongly dominated by $N\left(E_{F}\right)$, it is, however, conceivable that $T_{c}$ may be improved by doping. This paper is astep in the identification of HTCS at ambient pressure in light-element covalent metals using ab initio screening techniques.

\section{ACKNOWLEDGMENTS}

The authors would like to thank A.N. Kolmogorov for a thorough reading of our paper and useful suggestions. S.S., S.C., and W.L. acknowledge computational resources from the dCluster of the Graz University of Technology and the VSC3 of the Vienna University of Technology and support through the FWF, Austrian Science Fund, Project No. P30269-N36 (Superhydra). M.A. acknowledges support from the Swiss National Science Foundation (Projects No. P300P2158407, No. P300P2-174475, and No. P4P4P2-180669). L.B. acknowledges support from Fondo Ateneo Sapienza 201718 and computational resources from CINECA, project HiTSEPH.

\section{APPENDIX A: ELECTRON-PHONON SPECTRA OF THE SIXTEEN REPRESENTATIVE STRUCTURES}

\section{Electronic properties}

The total (black) and partial electronic DOS ( $\mathrm{C}$ in red and $\mathrm{B}$ in blue) of all the structures arranged by family are shown in Fig. 9 (D), Fig. 10 (G), Fig. 11 (S) and Fig. 12 (T).

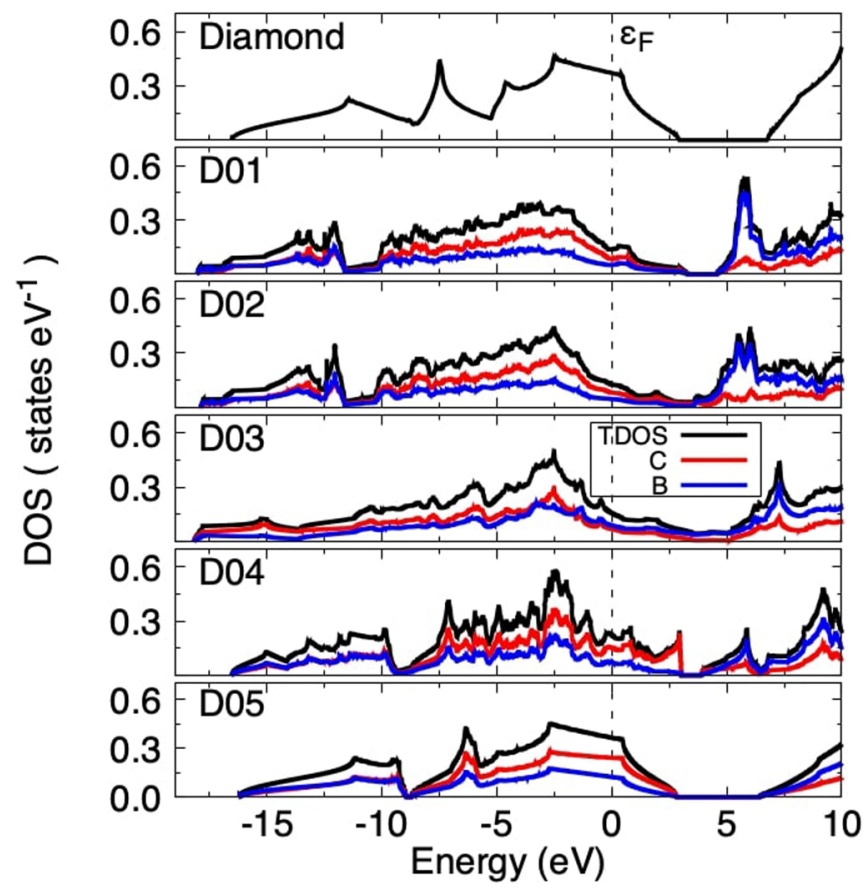

FIG. 9. The top panel shows the electronic DOS of the reference diamond structure and rest, the total DOS (black) and partial contribution of C (red) and B (blue) atoms in all the diamond structures.

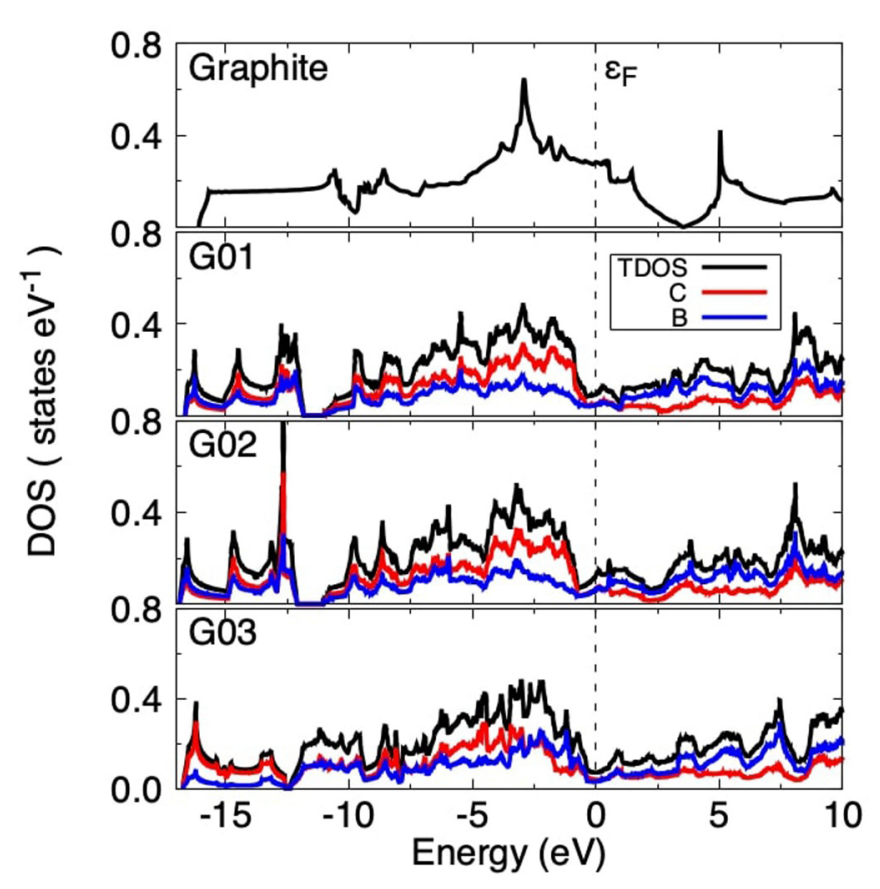

FIG. 10. The top panel shows the electronic DOS of the reference graphite structure and rest, the total DOS (black) and partial contribution of $\mathrm{C}$ (red) and $\mathrm{B}$ (blue) atoms in all the graphite structures.

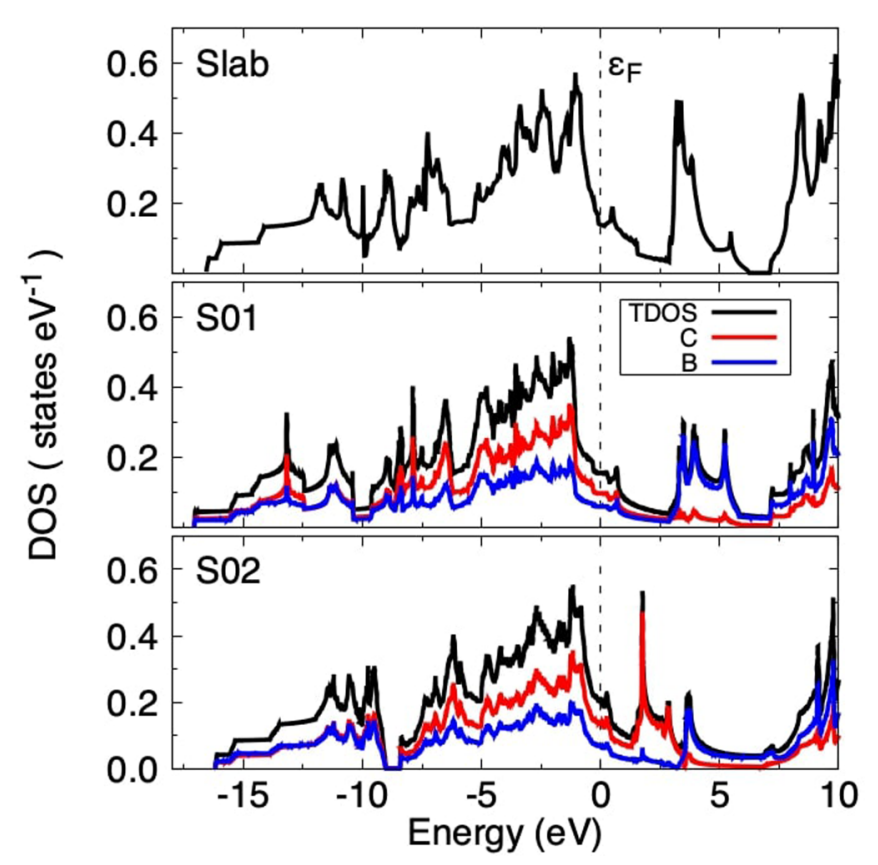

FIG. 11. The top panel shows the electronic DOS of the reference slab structure and rest, the total DOS (black) and partial contribution of $\mathrm{C}$ (red) and $\mathrm{B}$ (blue) atoms in all the slab structures. 


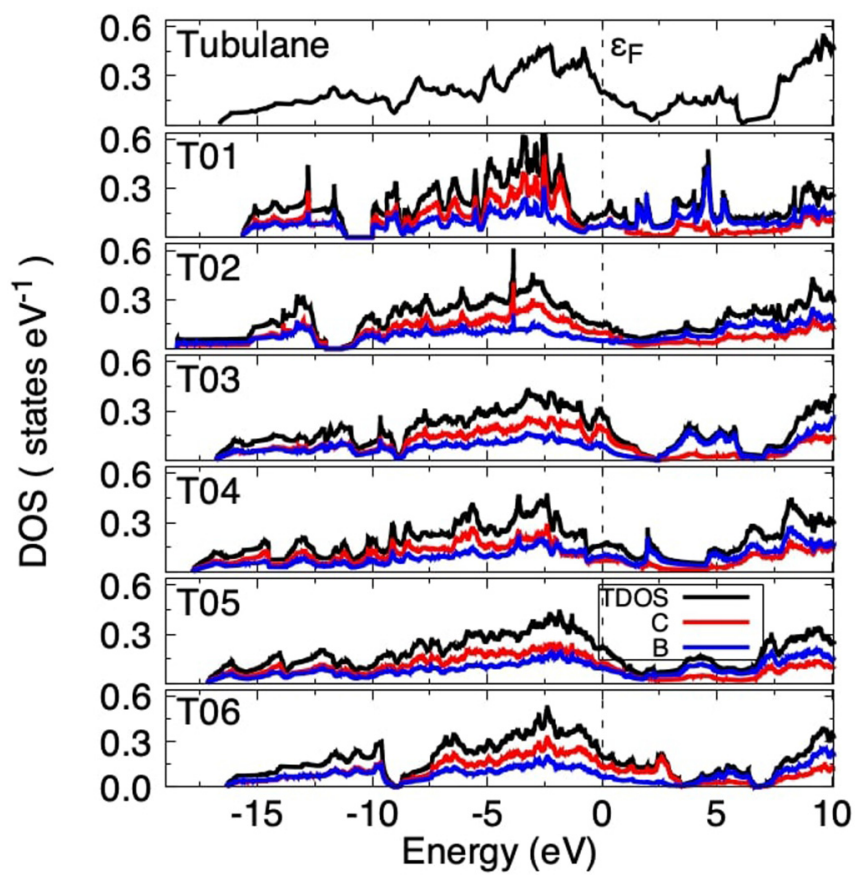

FIG. 12. The top panel shows the electronic DOS of the reference tubulane structure and rest, the total DOS (black) and partial contribution of $\mathrm{C}$ (red) and $\mathrm{B}$ (blue) atoms in all the tubulane structures.

\section{Vibrational properties}

The total (black) and partial phonon DOS ( $\mathrm{C}$ in red and $\mathrm{B}$ in blue) of all the structures arranged by family are shown in Fig. 13 (D), Fig. 14 (G), Fig. 15 (S), and Fig. 16 (T).

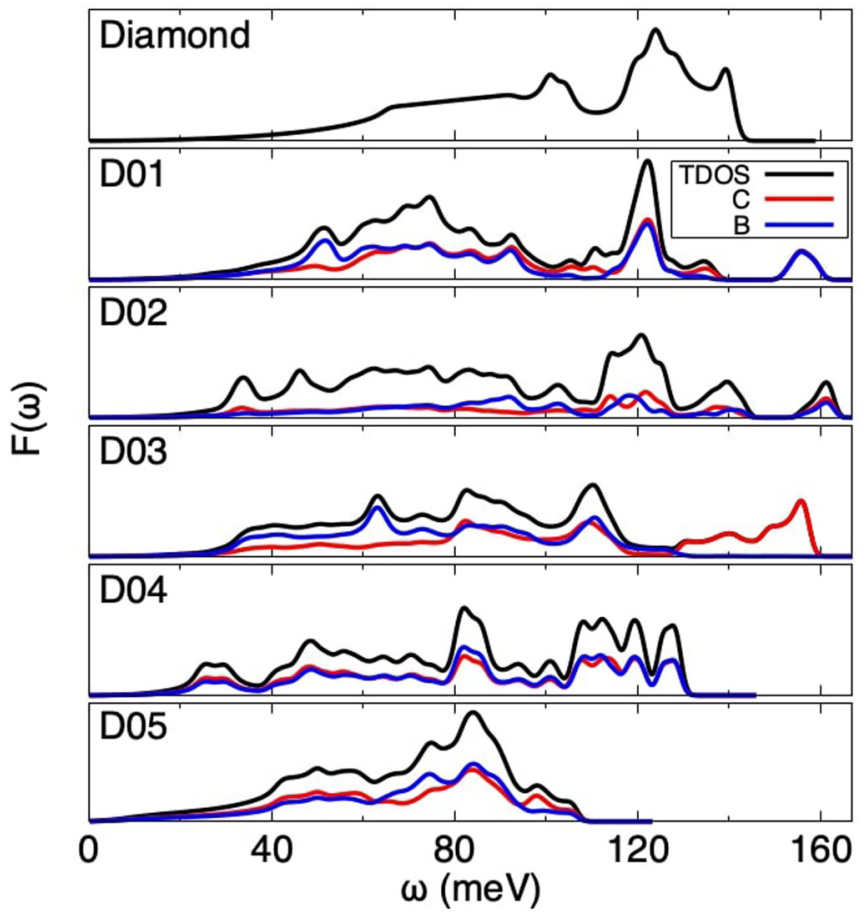

FIG. 13. The top panel shows the phonon DOS $F(\omega)$ of the reference diamond structure and rest, the total DOS (black) and partial contribution of $\mathrm{C}$ (red) and $\mathrm{B}$ (blue) atoms in all the diamond structures.

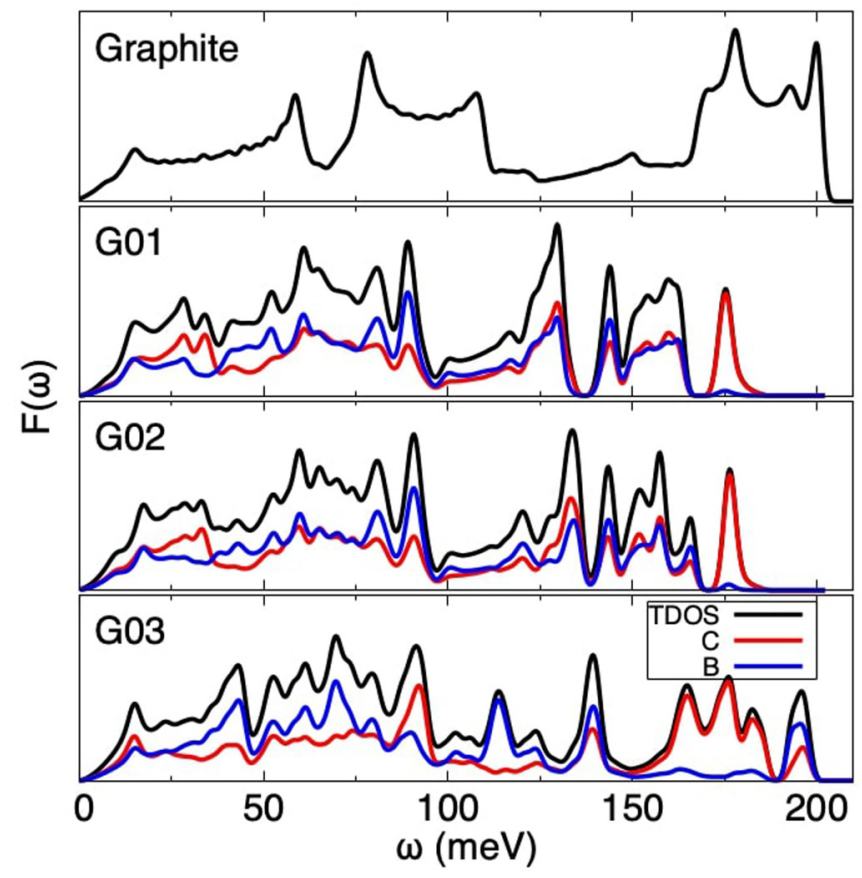

FIG. 14. The top panel shows the phonon DOS $F(\omega)$ of the reference graphite structure and rest, the total DOS (black) and partial contribution of C (red) and B (blue) atoms in all the graphite structures.

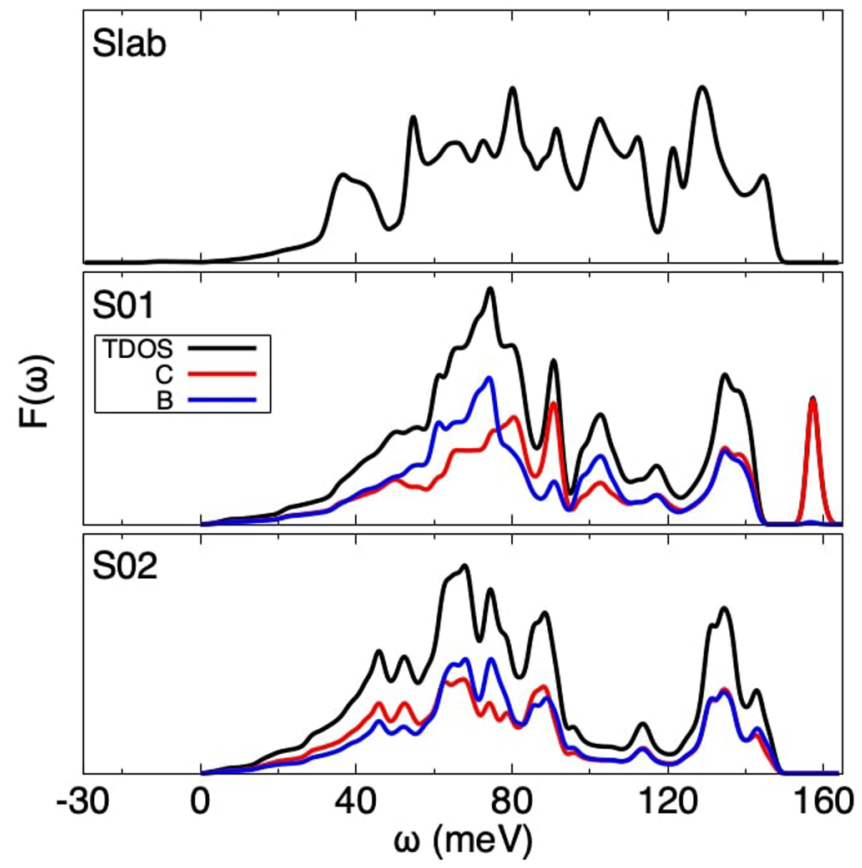

FIG. 15. The top panel shows the phonon DOS $F(\omega)$ of the reference slab structure and rest, the total DOS (black) and partial contribution of $\mathrm{C}$ (red) and B (blue) atoms in all the slab structures. 


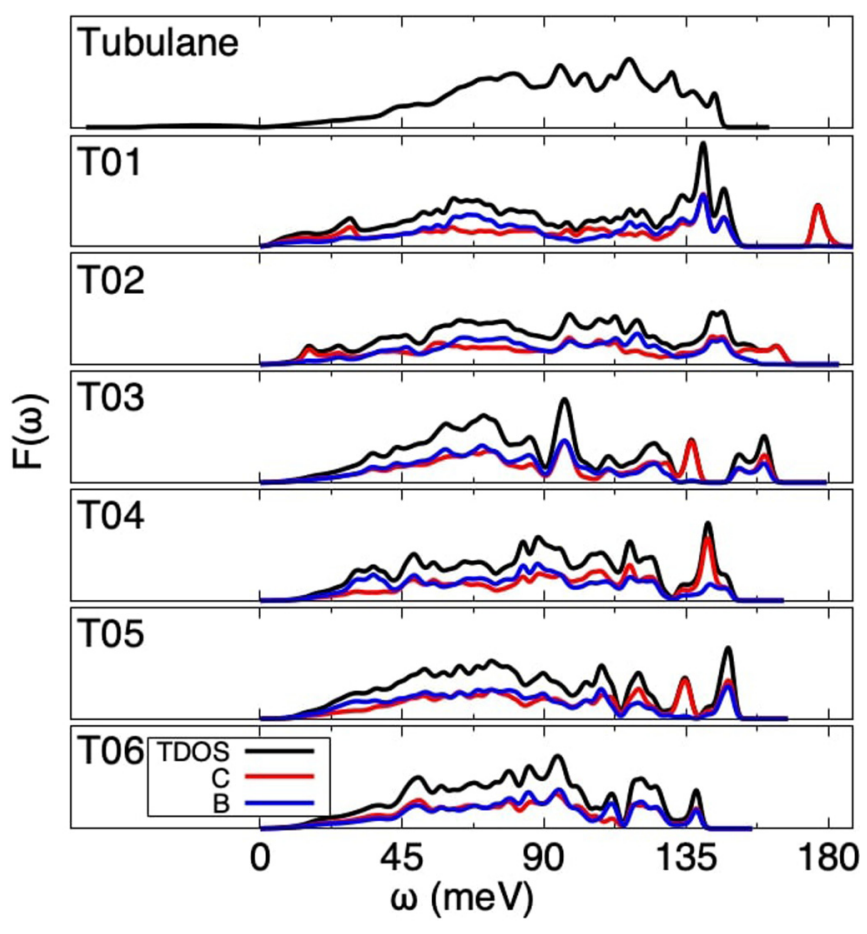

FIG. 16. The top panel shows the phonon DOS F $(\omega)$ of the reference tubulane structure and rest, the total DOS (black) and partial contribution of $\mathrm{C}$ (red) and $\mathrm{B}$ (blue) atoms in all the tubulane structures.

\section{Electron-phonon spectra}

The Eliashberg spectral function $\alpha^{2} \mathrm{~F}(\omega)$ (black lines) and ep coupling constant $\lambda(\omega)$ (dashed orange lines) of all the structures arranged by family are shown in Fig. 17 (D), Fig. 18 (G), Fig. 19 (S) and Fig. 20 (T).

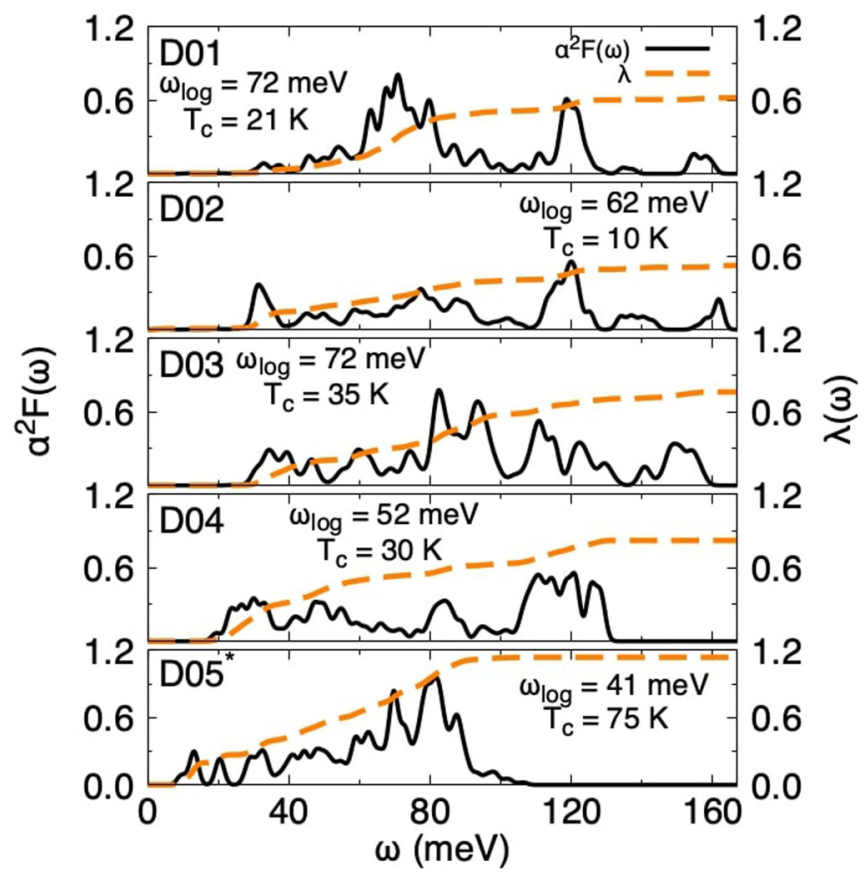

FIG. 17. The Eliashberg spectral function $\alpha^{2} \mathrm{~F}(\omega)$ and ep coupling constant $\lambda(\omega)$ of the diamond structures. The $\alpha^{2} \mathrm{~F}(\omega)$ and $\lambda(\omega)$ of D05 are scaled down by 0.5 .

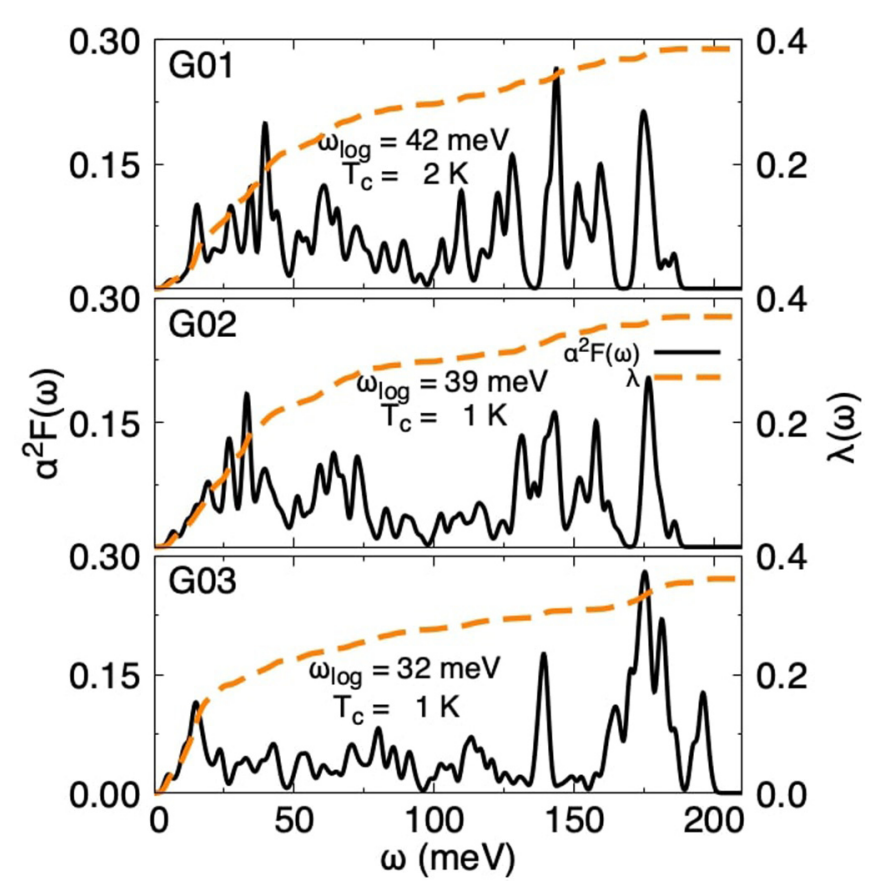

FIG. 18. The Eliashberg spectral function $\alpha^{2} \mathrm{~F}(\omega)$ and $e p$ coupling constant $\lambda(\omega)$ of the graphite structures.

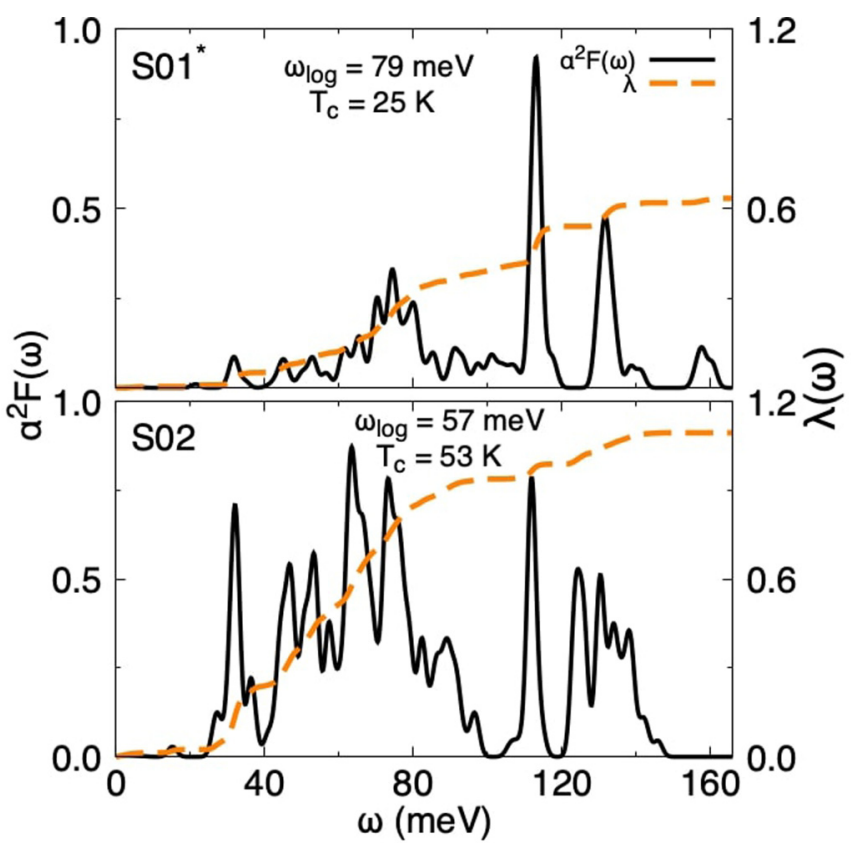

FIG. 19. The Eliashberg spectral function $\alpha^{2} \mathrm{~F}(\omega)$ and $e p$ coupling constant $\lambda(\omega)$ of the slab structures. The $\alpha^{2} \mathrm{~F}(\omega)$ of S01 is scaled down by 0.5 . 


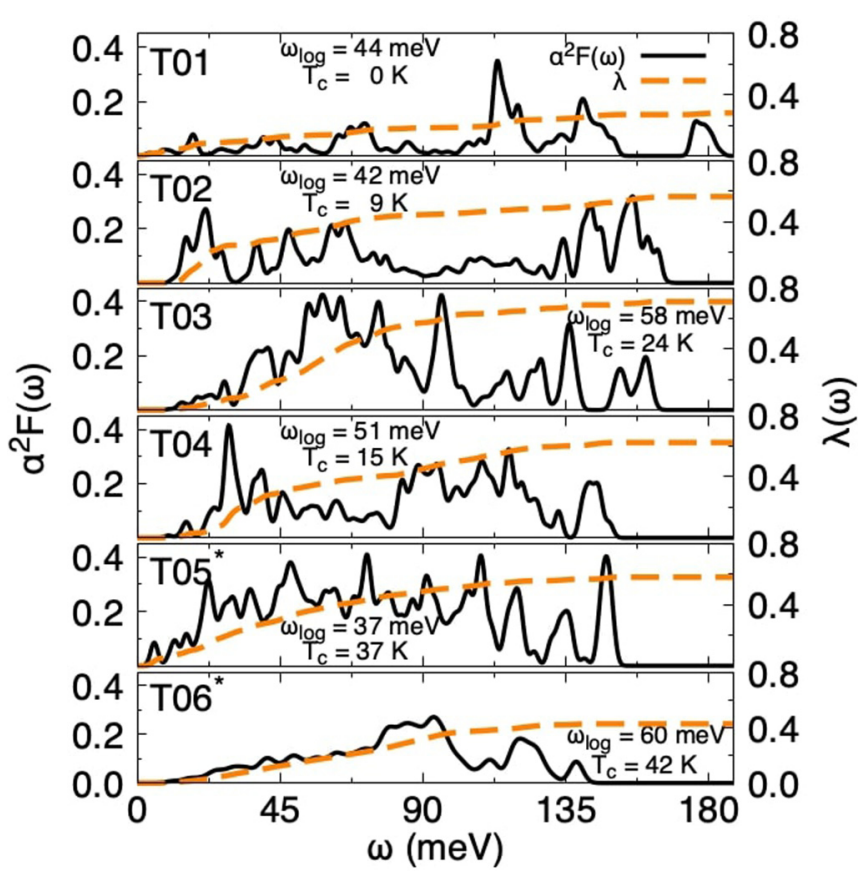

FIG. 20. The Eliashberg spectral function $\alpha^{2} \mathrm{~F}(\omega)$ and $e p$ coupling constant $\lambda(\omega)$ of the tubulane structures. The $\lambda(\omega)$ of T05 is scaled down by 0.5 . For T06, both the $\alpha^{2} \mathrm{~F}(\omega)$ and $\lambda(\omega)$ are scaled down by factor of 0.5 .

\section{APPENDIX B: COMPUTATIONAL METHODS}

\section{Structure prediction and DFT calculations}

The MH method [33-35,64,65] was used for an efficient scanning of the potential energy surface to find low-energy structures. The density functional theory (DFT) calculations for total-energy and relaxations were carried out using the Vienna Ab-Initio Simulation Package (VASP) [66,67]; $\mathrm{B}$ and $\mathrm{C}$ atoms were described by the built-in projector augmented-wave (PAW) potentials [68] with the PerdewBurke-Ernzerhof (PBE) exchange-correlation functional [69]. The energy cutoff used for the DFT runs was $380 \mathrm{eV}$, which ensures an accuracy of $\sim 10 \mathrm{meV} /$ atom.

The postrelaxation, energy evaluation, and calculation of the electronic DOS of all structures were performed using VASP with the same set of PAW potentials and PBE functional as used in the MH runs, but including van der Waals D3 dispersion corrections with Becke-Jonson damping [70]. A threshold of $1 \mathrm{meV} / \AA$ force of each atom and $0.1 \mathrm{kbar}$ on stress was set for the relaxation. The energy cutoff used for the postrelaxation calculation was $500 \mathrm{eV}$. For the relaxation and energy evaluation, the reciprocal (k) space integration employed a uniform $\mathbf{k}$ grid with a resolution of $2 \pi \times 0.10 \AA^{-1}$ centered at the $\Gamma$ point a Gaussian smearing of width $0.10 \mathrm{eV}$. For an accurate evaluation of the electronic DOS, we employed the improved tetrahedron method, as implemented in VASP [71].

The formation energy of all the BC structures has been evaluated with the formula

$$
\Delta E=\frac{E_{\mathrm{BC}}-n_{C} E_{C}-n_{B} E_{B}}{n_{C}+n_{B}},
$$

where $\mathrm{E}_{B C}$ is the total energy of the $\mathrm{BC}$ structure, $E_{C}$ and $E_{B}$ are the total energy per atom of the reference $\mathrm{C}$ and $\mathrm{B}$ structures and $\mathrm{n}_{C}$ and $\mathrm{n}_{B}$ are the total number of $\mathrm{C}$ and $\mathrm{B}$ atoms in the $\mathrm{BC}$ structure.

\section{Phonon and electron-phonon coupling calculations}

The phonon calculations at the $\Gamma$ point on the postrelaxed structures and the complete phonon calculations of phonon spectra and $e p$ matrix elements were carried out within DFPT, as implemented in the plane-wave pseudopotential code QUANTUM ESPRESSO 6.4.1 [72,73]. Atoms were described by optimized norm-conserving Vanderbilt (ONCV) pseudopotentials [74]. For the initial phonon calculations at the $\Gamma$ point on the large DB of structures, a PBE functional was used, whereas the remaining calculations were done with ONCV pseudopotentials [75] with Perdew-Wang92LDA functional [56], which ensures more accurate relaxations for layered structures. The $e p$ matrix calculations were carried out on regular $\Gamma$-centered Monkhorst-Pack $4 \times 4 \times 4$ grids for phonons $(\mathbf{q})$ and $8 \times 8 \times 8$ grids for electrons $(\mathbf{k})$. The selected structures were re-relaxed to a threshold force of $0.1 \mathrm{meV} / \AA$ and stress of $0.1 \mathrm{kbar}$ prior to phonon and ep; calculations with the LDA functional. For these calculations, an energy cutoff of 80 Ry was used with a Gaussian smearing of $0.04 \mathrm{Ry}$ for $\mathbf{k}$-space integration. For all structures, we employed an eight-atom supercell. In the case of the D05 structure, where symmetry allowed us to reduce the structure to two atoms/cell, a phonon grid of $q=8 \times 8 \times 8$ mesh was used.

The optimal choice of reciprocal space grids, the $e p$ coupling calculations, were fixed by performing extensive convergence tests on the D05 structure, which can be reduced to a two-atom primitive cell, the supercell of eight atoms of the $\mathrm{BC}$ diamond structure against the two-atom primitive unit cell of the BC diamond structure. For this cell, $\lambda$ and $\omega_{\log }$ were converged to within $5 \%$ for $\mathbf{q}(\mathbf{k})$ meshes $8(32) \times 8(32) \times$ $8(32)$ meshes in reciprocal space for electrons (phonons). The same accuracy is obtained with $4(8) \times 4(8) \times 4(8)$ grids in the eight-atom unit cell. The same parameters were then used for all structures.

\section{Screening protocol and structure selection}

In this paper, we have developed a three-stage protocol to identify superconductivity candidates from an initial pool of 320 metastable MH structures. In the first step, we wanted to prune out structures which had no potential for superconductivity. We thus needed to identify structures which should be (i) plausible to be realized in experimental conditions [47], (ii) metallic, (iii) dynamically stable, and (iv) exhibit stiff directional bonds, which ensure large phonon frequencies and $e p$ matrix elements.

Each of the these qualitative features can be estimated by the energy of formation $\Delta \mathrm{E}$ (i), electronic DOS at the Fermi level $\mathrm{N}\left(E_{F}\right)$ (ii), and the phonon spectrum (iii), respectively. The energy of formation and the electronic DOS were already calculated for all the 320 structures after the postrelaxation step.

For the dynamical stability, a full calculation of the phonon spectrum is too expensive to be feasible, 
whereas calculating the phonon frequencies $\omega_{i}$ only at the $\Gamma$ point is relatively inexpensive. Hence, we calculated the $\omega_{i}$ 's only at the $\Gamma$ point. This approach is not sufficient to assess the dynamical stability of the structure but it allowed us to reduce the pool of candidates, removing structures with imaginary $\omega_{i}$ 's.

From the $\omega_{i}$ 's, we further constructed a single-number descriptor $\omega_{\text {avg }}$, which is an average of all optical phonon frequencies at the $\Gamma$ point.

With these three quantities, i.e., $\Delta \mathrm{E}, \mathrm{N}\left(E_{F}\right)$ and $\omega_{\text {avg }}$, in hand, we then developed and used the following three-step screening process:

(i) Step 1: Structures with $\Delta E \leqslant 0.5 \mathrm{eV} /$ atom with respect to graphite and $\alpha$-rhombohedral- $\mathrm{B}_{12}$ are retained.

(ii) Step 2: Structures with $\mathrm{N}\left(E_{F}\right) \geqslant 0.1$ states/eV/atom are retained.

(iii) Step 3: Structures with $\mathrm{N}\left(E_{F}\right) \times \omega_{\text {avg }} \geqslant 0.02$ states/atom are retained.

These requirements are still broad enough that the initial subset was reduced to 116 candidates, which still constitute a too-large pool for a complete calculation of superconducting properties. To reduce the total pool to a manageable number, we hand picked a few structures (five to six) for each family (D, G, S, and T) from the final set of screened candidates. While selecting the structures, care was taken that they represent different values of $\mathrm{N}\left(E_{F}\right)$ and $\omega_{\text {avg }}$ values. For these structures, we performed full calculations of the phonon and $e p$ coupling spectra over the full BZ. If any of the initally selected structures was thus found to be dynamically unstable, it was replaced with another candidate with similar values of $\mathrm{N}\left(E_{F}\right)$ and $\omega_{\text {avg. }}$. The positions of the selected structures in the energy versus volume plot in Fig. 1 are shown by the dark colored symbols, whereas

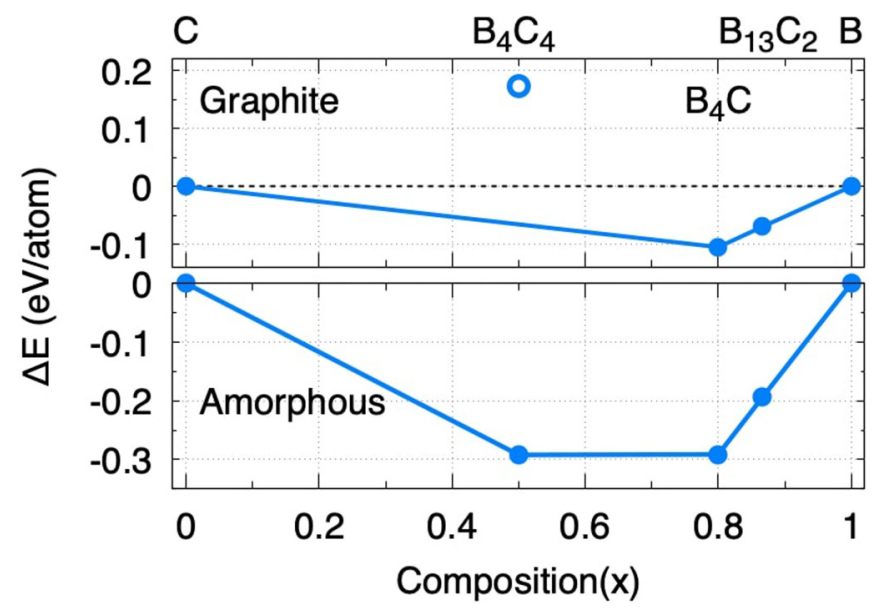

FIG. 21. Convex hull of the boron-carbon system with respect to graphite $\mathrm{C}$ and amorphous $\mathrm{C}$ in top and bottom panels, respectively. The reference for $\mathrm{B}$ is $\alpha-\mathrm{R}-\mathrm{B}_{12}$. The reference energy for the amorphous $\mathrm{C}$ is obtained from Ref. [47].

the rest of the structures are indicated by the light colored symbols.

\section{Convex hull}

Vibrational enthalpy has a negligible effect on the convex hull. An estimate using the linear approximation for the composition-volume-energy hull (NVE) [76] shows that the relative order of phases on the convex hull is also preserved for pressures in a $0-50 \mathrm{GPa}$ range.

The convex hull of the $\mathrm{BC}$ structures w.r.t. graphite and amorphous for carbon and $\alpha-\mathrm{R}-\mathrm{B}_{12}$ for boron is shown in Fig. 21
[1] N. W. Ashcroft, Phys. Rev. Lett. 21, 1748 (1968).

[2] V. L. Ginzburg, J. Stat. Phys. 1, 3 (1969).

[3] E. Wigner and H. B. Huntington, J. Chem. Phys. 3, 764 (1935).

[4] J. A. Flores-Livas, L. Boeri, A. Sanna, G. Profeta, R. Arita, and M. Eremets, Phys. Rep. (2020).

[5] M. I. Eremets, A. P. Drozdov, P. P. Kong, and H. Wang, Nat. Phys. 15, 1246 (2019).

[6] P. Loubeyre, F. Occelli, and P. Dumas, Nature 577, 631 (2020).

[7] N. W. Ashcroft, Phys. Rev. Lett. 92, 187002 (2004).

[8] D. Duan, Y. Liu, F. Tian, D. Li, X. Huang, Z. Zhao, H. Yu, B. Liu, W. Tian, and T. Cui, Sci. Rep. 4, 6968 (2014).

[9] A. Drozdov, M. Eremets, and I. Troyan, arXiv:1508.06224.

[10] H. Liu, I. I. Naumov, R. Hoffmann, N. Ashcroft, and R. J. Hemley, Proc. Natl. Acad. Sci. 114, 6990 (2017).

[11] M. Somayazulu, M. Ahart, A. K. Mishra, Z. M. Geballe, M. Baldini, Y. Meng, V. V. Struzhkin, and R. J. Hemley, Phys. Rev. Lett. 122, 027001 (2019).

[12] A. P. Drozdov, P. P. Kong, V. S. Minkov, S. P. Besedin, M. A. Kuzovnikov, S. Mozaffari, L. Balicas, F. F. Balakirev, D. E. Graf, V. B. Prakapenka, E. Greenberg, D. A. Knyazev, M. Tkacz, and M. I. Eremets, Nature 569, 528 (2019).

[13] F. Peng, Y. Sun, C. J. Pickard, R. J. Needs, Q. Wu, and Y. Ma, Phys. Rev. Lett. 119, 107001 (2017).
[14] L. Boeri and G. B. Bachelet, J. Phys.: Condens. Matter 31, 234002 (2019).

[15] J. Nagamatsu, N. Nakagawa, T. Muranaka, Y. Zenitani, and J. Akimitsu, Nature 410, 63 (2001).

[16] J. M. An and W. E. Pickett, Phys. Rev. Lett. 86, 4366 (2001).

[17] E. Ekimov, V. Sidorov, E. Bauer, N. Mel'Nik, N. Curro, J. Thompson, and S. Stishov, Nature 428, 542 (2004).

[18] L. Boeri, J. Kortus, and O. K. Andersen, Phys. Rev. Lett. 93, 237002 (2004).

[19] K.-W. Lee and W. E. Pickett, Phys. Rev. Lett. 93, 237003 (2004).

[20] X. Blase, C. Adessi, and D. Connétable, Phys. Rev. Lett. 93, 237004 (2004).

[21] F. Giustino, J. R. Yates, I. Souza, M. L. Cohen, and S. G. Louie, Phys. Rev. Lett. 98, 047005 (2007).

[22] H. Rosner, A. Kitaigorodsky, and W. E. Pickett, Phys. Rev. Lett. 88, 127001 (2002).

[23] G. Savini, A. C. Ferrari, and F. Giustino, Phys. Rev. Lett. 105, 037002 (2010).

[24] A. N. Kolmogorov and S. Curtarolo, Phys. Rev. B 73, 180501(R) (2006).

[25] A. N. Kolmogorov, S. Hajinazar, C. Angyal, V. L. Kuznetsov, and A. P. Jephcoat, Phys. Rev. B 92, 144110 (2015). 
[26] A. Y. Liu and I. I. Mazin, Phys. Rev. B 75, 064510 (2007).

[27] K. Alberi, M. B. Nardelli, A. Zakutayev, L. Mitas, S. Curtarolo, A. Jain, M. Fornari, N. Marzari, I. Takeuchi, M. L. Green et al., J. Phys. D: Appl. Phys. 52, 013001 (2018).

[28] G. R. Schleder, A. C. M. Padilha, C. M. Acosta, M. Costa, and A. Fazzio, J. Phys.: Mater. 2, 032001 (2019).

[29] V. Stanev, C. Oses, A. G. Kusne, E. Rodriguez, J. Paglione, S. Curtarolo, and I. Takeuchi, npj Comput. Mater. 4, 1 (2018).

[30] T. Ishikawa, T. Miyake, and K. Shimizu, Phys. Rev. B 100, 174506 (2019).

[31] S. R. Xie, G. R. Stewart, J. J. Hamlin, P. J. Hirschfeld, and R. G. Hennig, Phys. Rev. B 100, 174513 (2019).

[32] M. J. Hutcheon, A. M. Shipley, and R. J. Needs, Phys. Rev. B 101, 144505 (2020).

[33] S. Goedecker, J. Chem. Phys. 120, 9911 (2004).

[34] S. Goedecker, W. Hellmann, and T. Lenosky, Phys. Rev. Lett. 95, 055501 (2005).

[35] M. Amsler and S. Goedecker, J. Chem. Phys. 133, 224104 (2010).

[36] A. Jay, O. Hardouin Duparc, J. Sjakste, and N. Vast, J. Appl. Phys. 125, 185902 (2019).

[37] M. Calandra, N. Vast, and F. Mauri, Phys. Rev. B 69, 224505 (2004).

[38] M. Calandra and F. Mauri, Phys. Rev. Lett. 101, 016401 (2008).

[39] J. E. Moussa and M. L. Cohen, Phys. Rev. B 77, 064518 (2008).

[40] J. E. Moussa and M. L. Cohen, Phys. Rev. B 74, 094520 (2006).

[41] J. E. Moussa and M. L. Cohen, Phys. Rev. B 78, 064502 (2008).

[42] A. Bhaumik, R. Sachan, S. Gupta, and J. Narayan, ACS Nano 11, 11915 (2017).

[43] A. Bhaumik, R. Sachan, and J. Narayan, ACS Nano 11, 5351 (2017).

[44] A. Bhaumik, R. Sachan, and J. Narayan, J. Appl. Phys. 122, 045301 (2017).

[45] Y. Sakai, J. R. Chelikowsky, and M. L. Cohen, Phys. Rev. Mater. 3, 084802 (2019).

[46] W. L. McMillan, Phys. Rev. 167, 331 (1968).

[47] M. Aykol, S. S. Dwaraknath, W. Sun, and K. A. Persson, Sci. Adv. 4, eaaq0148 (2018).

[48] Y. Lin, Z. Zhao, T. A. Strobel, and R. E. Cohen, Phys. Rev. B 94, 245422 (2016).

[49] X. Jiang, J. Zhao, Y.-L. Li, and R. Ahuja, Adv. Funct. Mater. 23, 5846 (2013).

[50] X. Feng, Q. Wu, Y. Cheng, B. Wen, Q. Wang, Y. Kawazoe, and P. Jena, Carbon 127, 527 (2018).

[51] Y. Chen, Y. Xie, S. A. Yang, H. Pan, F. Zhang, M. L. Cohen, and S. Zhang, Nano Lett. 15, 6974 (2015).

[52] S. Wang, D. Wu, B. Yang, E. Ruckenstein, and H. Chen, Nanoscale 10, 2748 (2018).

[53] Y. Gao, Y. Chen, Y. Xie, P.-Y. Chang, M. L. Cohen, and S. Zhang, Phys. Rev. B 97, 121108(R) (2018).
[54] Y. Chen, Y. Xie, Y. Gao, P.-Y. Chang, S. Zhang, and D. Vanderbilt, Phys. Rev. Mater. 2, 044205 (2018).

[55] R. Baughman and D. Galvao, Chem. Phys. Lett. 211, 110 (1993).

[56] J. P. Perdew and Y. Wang, Phys. Rev. B 45, 13244 (1992).

[57] See Supplemental Material at http://link.aps.org/supplemental/ 10.1103/PhysRevB.102.024519 for the CIF files of the 16 representative $\mathrm{BC}$ structures used for superconductivity studies.

[58] The threshold of distance for the presence of $\mathrm{C}-\mathrm{C}, \mathrm{B}-\mathrm{B}$, and B-C bonds are $\mathrm{d}_{\mathrm{CC}} \leqslant 1.5 \AA, \mathrm{d}_{\mathrm{BB}} \leqslant 1.85 \AA$, and $\mathrm{d}_{\mathrm{BC}} \leqslant 1.65 \AA$, respectively.

[59] J. Zhou, Q. Sun, Q. Wang, and P. Jena, Phys. Rev. B 92, 064505 (2015).

[60] S. Mann, P. Rani, R. Kumar, G. S. Dubey, and V. Jindal, RSC Adv. 6, 12158 (2016).

[61] The vibrational frequencies are calculated for the pure diamond structure with the lattice constant of D05, i.e., $a=3.74 \AA$, which is a $\sim 5 \%$ larger than the experimental lattice constant of diamond.

[62] G. Eliashberg, Sov. Phys. JETP 11, 696 (1960).

[63] J. J. Hopfield, Phys. Rev. 186, 443 (1969).

[64] M. Amsler, Minima hopping method for predicting complex structures and chemical reaction pathways, in Handbook of Materials Modeling: Applications: Current and Emerging Materials, edited by W. Andreoni and S. Yip (Springer International Publishing, Cham, 2018), pp. 1-20.

[65] M. Amsler, S. Rostami, H. Tahmasbi, E. R. Khajehpasha, S. Faraji, R. Rasoulkhani, and S. A. Ghasemi, Comp. Phys. Commun. 256, 107415 (2020).

[66] G. Kresse and F. J., Comput. Mat. Sci. 6, 15 (1996).

[67] G. Kresse and J. Furthmüller, Phys. Rev. B 54, 11169 (1996).

[68] G. Kresse and D. Joubert, Phys. Rev. B 59, 1758 (1999).

[69] J. P. Perdew, K. Burke, and M. Ernzerhof, Phys. Rev. Lett. 77, 3865 (1996).

[70] S. Grimme, S. Ehrlich, and L. Goerigk, J. Comput. Chem. 32, 1456 (2011).

[71] P. E. Blöchl, O. Jepsen, and O. K. Andersen, Phys. Rev. B 49, 16223 (1994).

[72] P. Giannozzi, O. Andreussi, T. Brumme, O. Bunau, M. B. Nardelli, M. Calandra, R. Car, C. Cavazzoni, D. Ceresoli, M. Cococcioni et al., J. Phys.: Condens. Matter 29, 465901 (2017).

[73] P. Giannozzi, S. Baroni, N. Bonini, M. Calandra, R. Car, C. Cavazzoni, D. Ceresoli, G. L. Chiarotti, M. Cococcioni, I. Dabo et al., J. Phys.: Condens. Matter 21, 395502 (2009).

[74] D. R. Hamann, Phys. Rev. B 88, 085117 (2013).

[75] M. Van Setten, M. Giantomassi, E. Bousquet, M. J. Verstraete, D. R. Hamann, X. Gonze, and G.-M. Rignanese, Comput. Phys. Commun. 226, 39 (2018).

[76] M. Amsler, V. I. Hegde, S. D. Jacobsen, and C. Wolverton, Phys. Rev. X 8, 041021 (2018). 